\title{
Naturlandschaft und Wandel der Kulturlandschaft im unteren und mittleren Einzugsgebiet der Emme
}

gezeigt an den Beispielen Grafenried (tieferes Mittelland), Heimiswil (Übergangszone), Heimisbach ("Dürrgraben», höheres Mittelland) und Rüderswilschachen-Rüegsauschachen (Schachensiedlungen)

Klaus Aerni

Itinerar und Zeitplan:

7.30 Abfahrt in Solothurn

8.00-9.15 Grafenried: Natur- und Kulturlandschaftsgenese, Betriebsbesichtigung (Herr Danuser)

9.45-10.05 Unter-Heimiswil: Naturlandschaft, ältere Besiedlung (Flurnamen)

10.15-10.30 Kaltacker: Naturlandschaft, Frage nach dem Beginn der Dreizelgenwirtschaft, moderne Betriebsformen der Landwirtschaft

10.35-11.00 Heimismatt: Rodungen und Hofteilungen, Auflösung der Dreizelgenwirtschaft im Weiler Ober-Heimiswil, Probleme der modernen Kulturlandschaft

12.40 Abfahrt von der Lueg (Mittagessen) über Affoltern-Grünen nach Tal

13.00-15.40 Wanderung durch den Heimisbach auf die Sparrenegg

13.30-14.15 Hof Hinter Liechtguet (Herr Gfeller): Betriebsbesichtigung, Probleme der Gemeinde Trachselwald

14.45-15.00 Hof Nülli: Natur- und Kulturlandschaftsgenese von Heimisbach, Betriebsform der Berghöfe Zueguet: Geburtshaus von Simon Gfeller

15.40 Abfahrt von der Sparrenegg über Wasen-Grünen nach Ramsei

16.00-16.40 Schnätzischachen bei Rüderswil: Entwicklung der Schachensiedlung im Emmental, Bauern und Tauner, die Verbauungen an der Emme

17.00-17.15 Rüegsauschachen: Moderne Entwicklung der Schachensiedlung, Zusammenfassung

18.00 Ankunft in Solothurn

\section{Einleitung}

Am Vorabend der Exkursion orientierte der Berichterstatter über das Exkursionsgebiet und charakterisierte Naturgrundlagen, politische Geschichte und Wandlungen der Kulturlandschaft in historischer Zeit. Die Ausführungen wurden ergänzt durch Herrn Markus Flückiger, Rapperswil, der seine Studien über frühneuzeitliche Veränderungen der Kulturlandschaft des Rapperswiler Plateaus erläuterte.

Ich konzentriere mich in der Berichterstattung auf einige wesentliche Punkte zum Thema des Landschaftswandels und verweise im übrigen auf die Literatur. Als Anregung für weiterführende Betrachtungen sind in den graphischen Figuren neben den auf der Exkursion besuchten Gemeinden auch noch das seeländische Dotzigen und das oberemmentalische Schangnau dargestellt.

Herrn Prof. B. Messerli und Herrn Staatsarchivar F. Häusler danke ich herzlich für Anregungen und Bemerkungen bei der Durchsicht des Manuskriptes.

\section{Grafenried - Tieferes Mittelland}

Naturlandschaftlich liegt Grafenried im Bereich der Aquitan-Mergel und -Sandsteine, die würmeiszeitlich überformt und mit Grundmoräne überlagert worden sind. Die Neigungswinkel betragen weniger als $10 \%$. Braunerden überdecken die flachen Kuppen, in kleinen Mulden finden sich Niedermoorböden.

Siedlungsgeschichtlich datieren die ältesten Funde aus der Hallstattzeit1. Aus römischer Zeit stammt der berühmte Münzfund zwischen Grafenried und Kernenried2. Die Flurbezeichnung Villenacher könnte auf eine römische Siedlung hindeuten. Über die Landnahme durch die Alemannen ist nichts Näheres bekannt. Sie dürfte nach Moosbrugger und Zinsli vor 700 erfolgt sein ${ }^{3}$.

Der Ort ist 1258 erstmals als "Gravenriet» erwähnt und gehörte damals den Grafen von Tierstein. 1262 verkaufte Rudolf von Tierstein seinen gesamten dortigen Besitz dem Frauenkloster Fraubrunnen. Nach der Reformation übernahm die Stadt Bern 1528 den Klosterbesitz und teilte Grafenried der Landvogtei Fraubrunnen zu4 4 . Dorfbrände von 1850 und 1915/16 haben das Dorfbild wesentlich umgestaltet ${ }^{1}$.

Die frühgeschichtliche und frühmittelalterliche 
Fig. 1 Übersicht

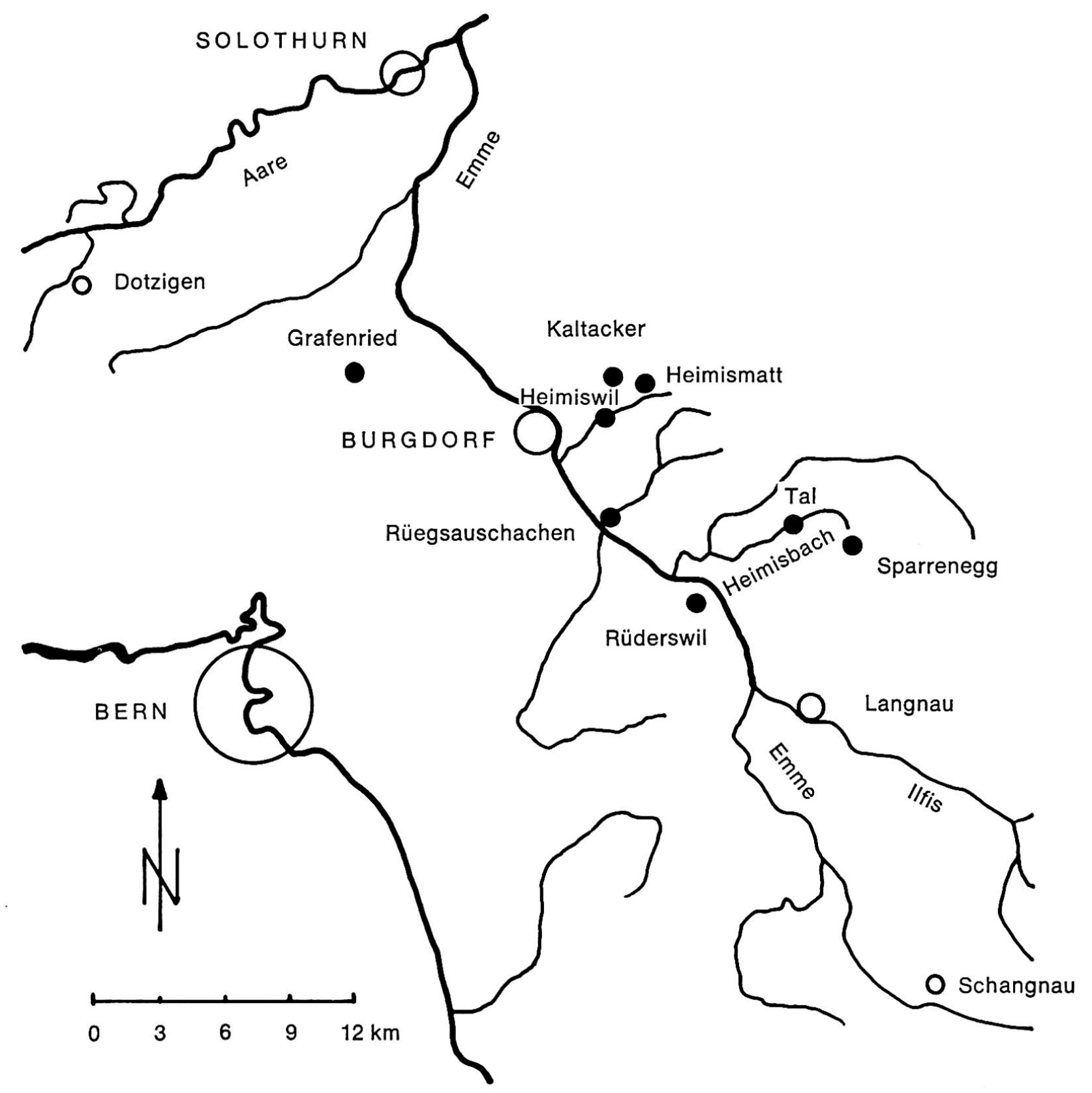

Flurverfassung von Grafenried ist nicht bekannt. Dagegen ist die seitherige Entwicklung in einzigartiger Weise in der Untersuchung P. Zryds über "Grafenried zur Zeit der Dreifelderwirtschaft» aufgehellt. Dank dieser Arbeit kann Grafenried als kulturlandschaftliche Typlokalität des tieferen Mittellandes bezeichnet werden. Es gelang Zryd auf
Grund verschiedener Urbarien die Geschichte jeder einzelnen Parzelle seit 1531 nachzuzeichnen.

Uber das Prinzip der Dreifelderwirtschaft im bernischen Bereich orientierten in neuerer Zeit Pulver (1956) und Häusler (1968). Eine ernsthafte Durchlöcherung der Dreizelgenwirtschaft, deren Hauptziel die Getreideproduktion war ${ }^{5}$, begann sich im 
Fig. 2 Erwerbsstruktur ausgewählter Gemeinden

a) absolute Darstellung
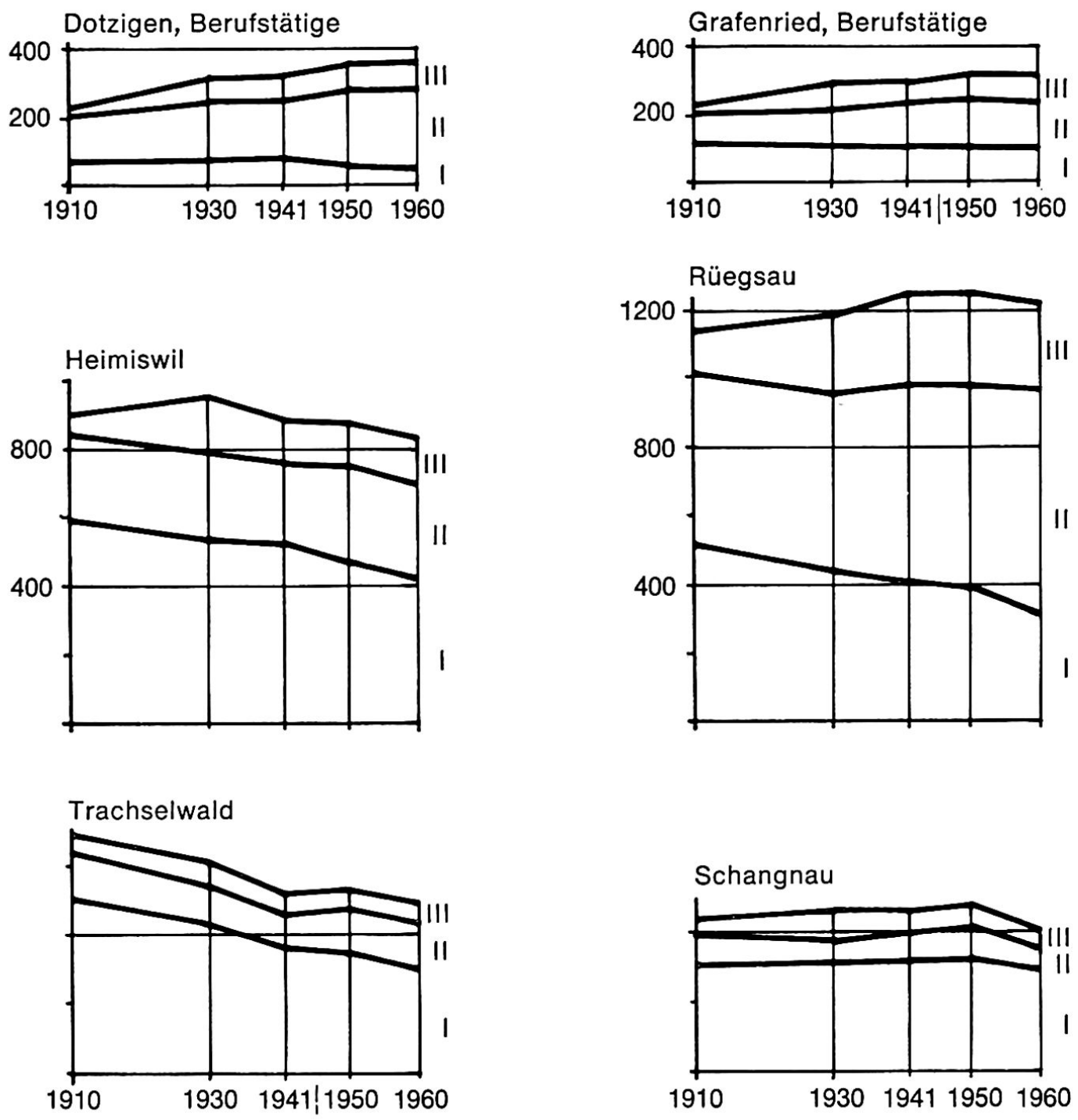

b) relative Darstellung in Dreieckskoordinaten (1910—1930-1950-1960) LANDWIRTSCHAFT (I)

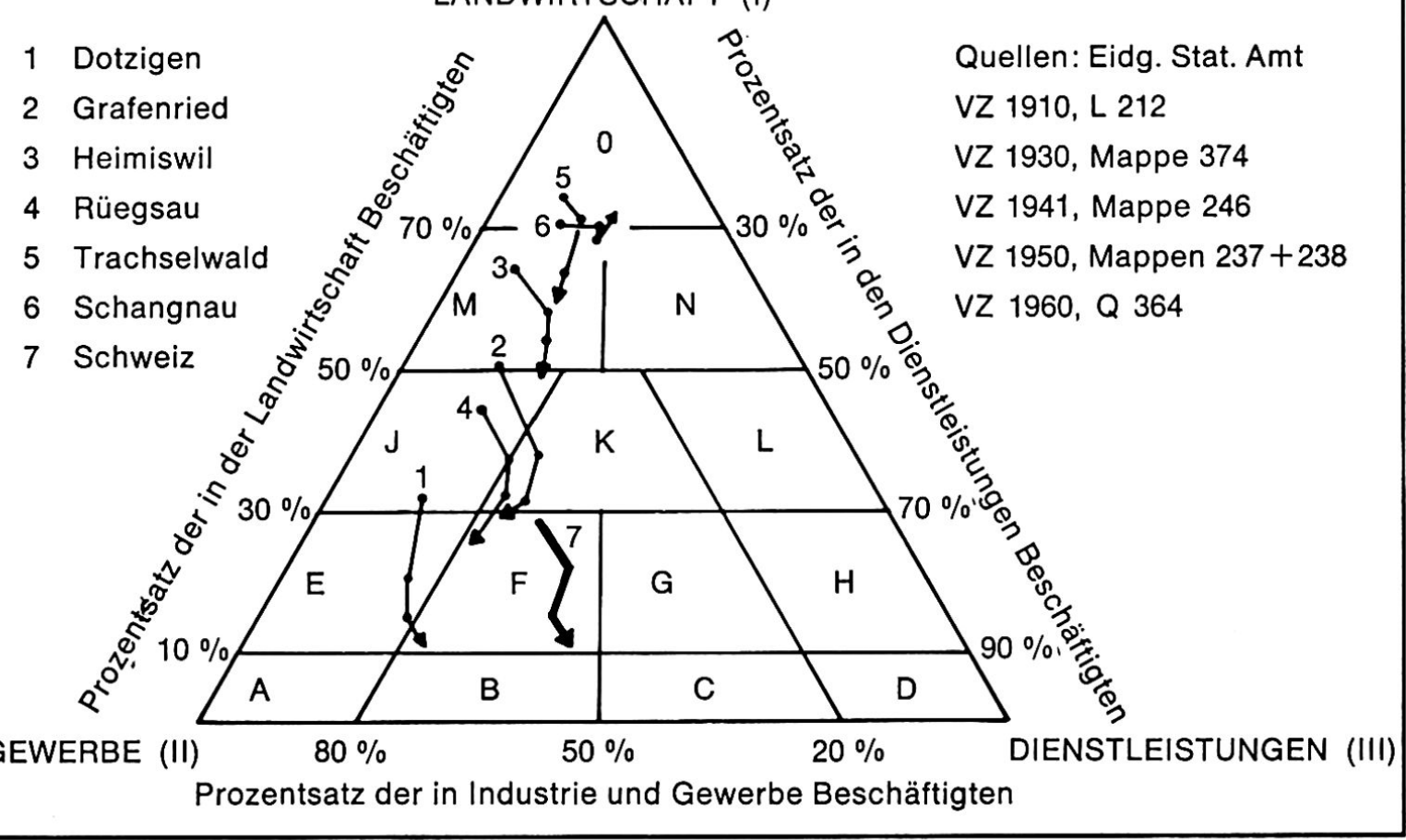


Fig. 3 Ausgewählte Wirtschaftsdaten I

Dotzigen Grafenried

a) Landwirtschaftliche Produktion
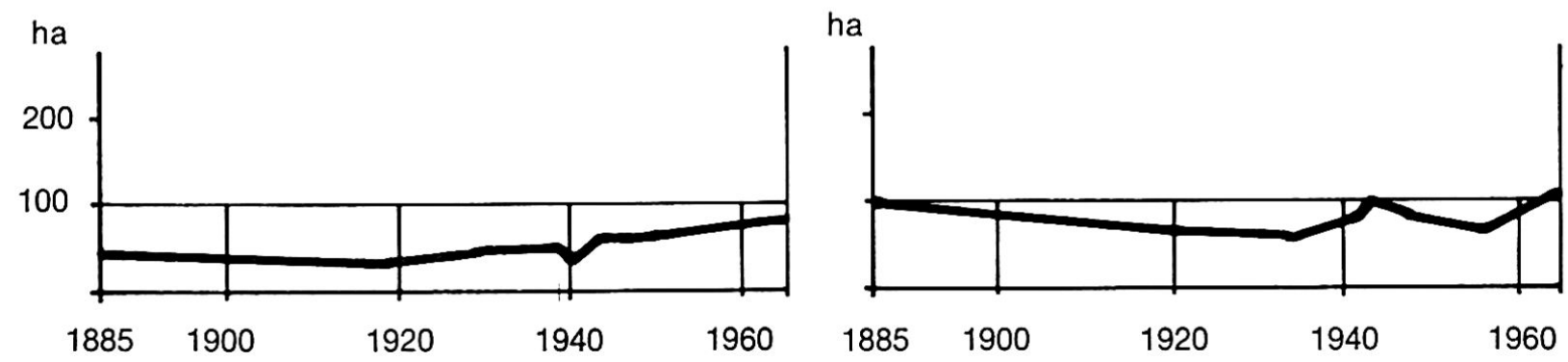

Getreidebau (Quellen: Publikationen Eidg. Stat. Amt, siehe Lit.-Verz.)
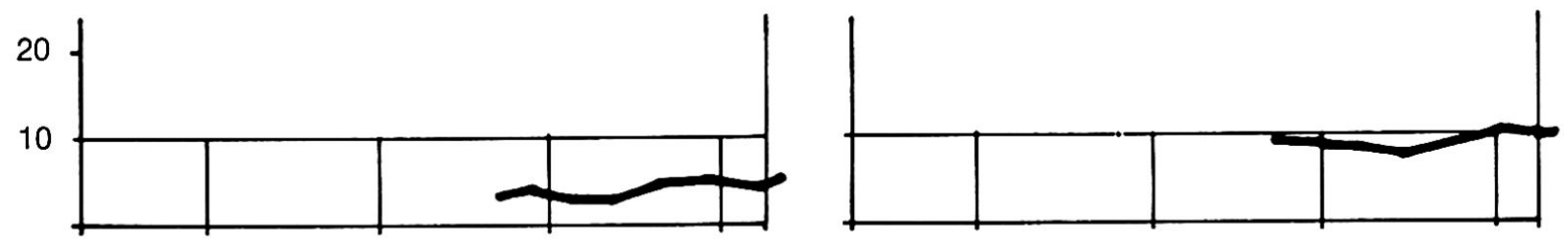

Milchproduktion in $1000 \mathrm{q} \quad$ (Quellen: Jahresberichte Milchverband Bern)
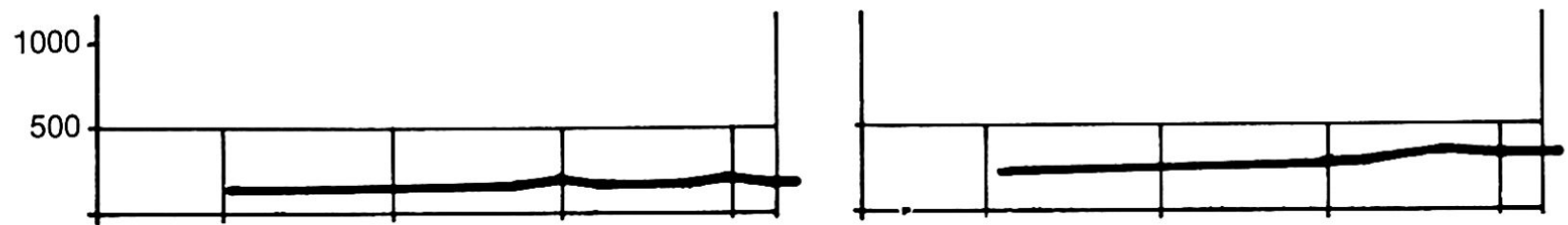

Milchkühe (Quelle: Landwirtschaftlicher Produktionskataster)

b) Struktur der Landwirtschaftsbetriebe

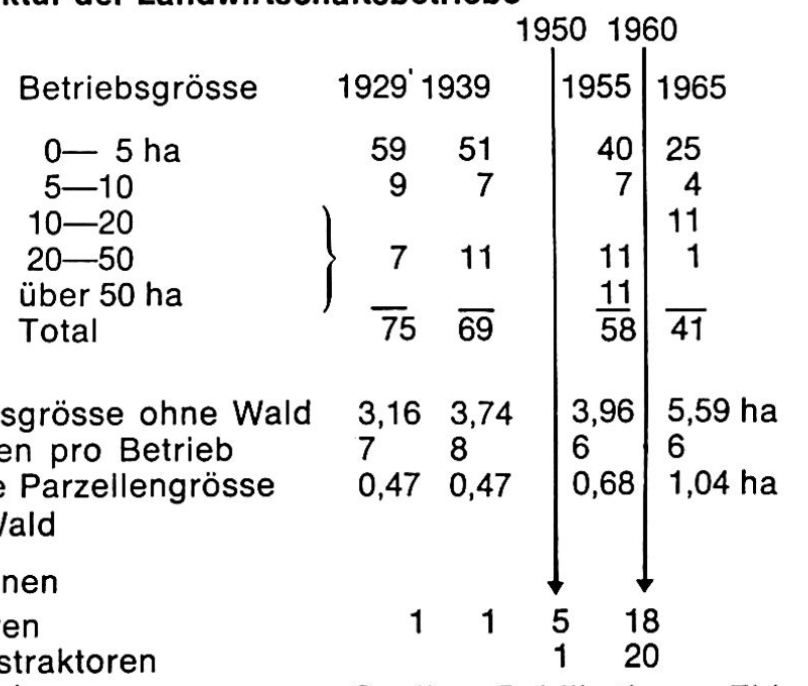

Quellen: Publikationen Eidg. Stat. Amt

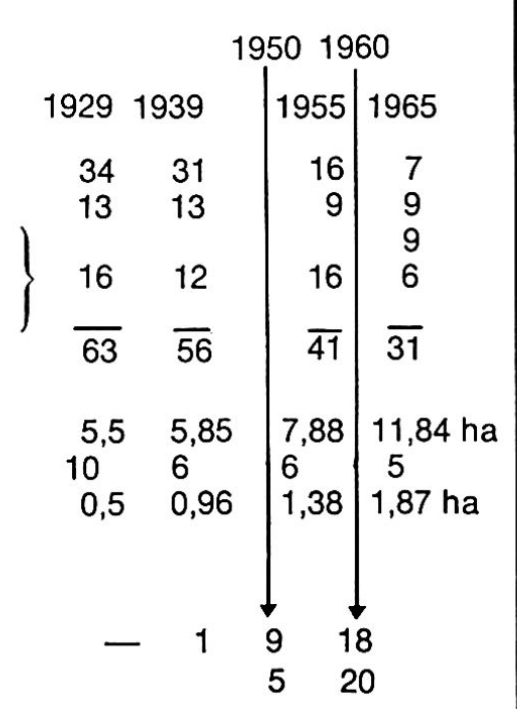

Einachstraktoren

Seilwinden

c) Industrie-, Gewerbe- und Dienstleistungsbetriebe

(1. Zahl = Betriebe, 2. Zahl = Beschäftigte)

sekundärer Sektor

19551965

22-117 23-98

$13-31 \quad 11-45$

tertiärer Sektor

$\overline{35}-\overline{148} \quad \overline{35}-\overline{143}$

Industriebetriebe mit $<20 \mathrm{~B}$.

20-99 Beschäftigte

(Quellen: Publikationen Eidg. Stat. Amt) 
tieferen bernischen Mittelland um die Mitte des 18. Jahrhunderts durch die Einführung des Kartoffelbaues auf der Brache, oft auch in den Getreidezelgen, abzuzeichnen ${ }^{6}$. Bestimmend für die weitere Entwicklung war dann das «Gutachten über die Brachfelder», das die vom Großen Rat eingesetzte "Landesökonomiekommission" in den Jahren 1764/65 abgab $^{7}$. 1780 äußerte sich Niklaus Emanuel Tscharner, der Präsident der 1759 gegründeten Ökonomischen Gesellschaft, wie folgt: «Zu dieser Zeit war im Kanton Bern, besonders im Mittelland, die Kartoffel als Hackfrucht allgemein angebaut, die alte Dreifelderwirtschaft mit nackter Brache größtenteils abgeschafft, statt deren entweder die veredelte Dreifelderwirtschaft oder die Graswirtschaft eingeführt, Stallfütterung, Klee- und Kunstgrasbau sowie die Anwendung des Gypses und Mergels allgemein bekannt und durchgeführt, die Allmenden entweder verteilt oder angebaut, der Landzins durchgehend um die Hälfte gestiegen ${ }^{8}$.»

Mit diesen Verbesserungen war die Landwirtschaft des tieferen Mittellandes auf die Umstellung zur Milchwirtschaft, die im höheren Mitelland bereits begonnen hatte, vorbereitet. Vom Ende des 17. Jahrhunderts an hatte nämlich die Ausfuhr von Alpenkäse eine immer größere Bedeutung erlangt ${ }^{9}$. Die Ökonomische Gesellschaft beschäftigte sich von 1762 an intensiv mit den Fragen der Käseprodukion und des Käsehandels. Die allgemeine Nachfrage des Auslandes förderte die Ausdehnung der Milchwirtschaft.

Die Frage stellte sich, ob neben den Alpbetrieben auch in den Talgebieten Exportkäse herstellbar sei. Entsprechende vortastende Versuche wurden in Genf, in der Waadt, in Freiburg, Luzern und am linken Zürichseeufer unternommen ${ }^{10}$. Bekannter sind die Daten aus dem Bernbiet: 1802 errichtete Emanuel Fellenberg in Hofwil eine Käserei und eine milchwirtschaftliche Versuchsstation ${ }^{11}$. 1815 gründete Rudolf Emanuel von Effinger-Wildegg in Kiesen eine Talkäserei; 1825 folgte jene von Aarwangen 12. Die Zeit war reif für die Umstellung, und damit bildete sich die alte Dreifelderwirtschaft rasch und endgültig zurück: zwischen 1830 und 1847 stieg die Zahl der Talkäsereien im Bernbiet von 15 auf 380, daneben bestanden noch 633 Alpkäsereien13. - In Grafenried wurde 1844 in einem Ofenhaus eine behelfsmäßig untergebrachte Käserei errichtet, an der sich zunächst 11 Lieferanten beteiligten. 1847 entstand dann ein Käsereigebäude ${ }^{14}$.

Die Umstellung in der Landwirtschaft erfaßte auch den Wald. Zur mittelalterlichen Wirtschaftsweise gehörte es, den Wald als Weide zu nutzen. In den tiefsten Lagen des Mittellandes war die Eiche im Mittelalter und in der frühen Neuzeit die Hauptholzart und diente der Landwirtschaft als Fruchtbaum für die Schweine15. Das Recht der Waldnutzung als Weide für die Schweine hieß Acherum und war durch verschiedenste Vorschriften geregelt ${ }^{16}$. Fassen wir die Veränderungen im Waldbild des tieferen Mittellandes nach Meyer zusammen 17: Mit der allgemeinen Verbreitung der Kartoffel um die Mitte des 18. Jahrhunderts, deren Abfälle von nun an als Schweinefutter dienten und die Eicheln ersetzten, begann die Eiche ihre Bedeutung zu verlieren. Die langsam wachsenden Bäume wurden nicht mehr nachgezogen; die Bestände überalterten. In der Mitte des 19. Jahrhunderts folgte ein zweiter Schlag, der sie weithin zum Verschwinden brachte: das Eisenbahnzeitalter rückte heran, die Schwellen dazu hatten die Eichen zu liefern. "50 Jahre Schwellenlieferung haben der Eiche mehr geschadet als 5000 Jahre Mastnutzung ${ }^{18}$.» Die entstandenen Kahlschlagflächen wurden mit Fichtenwald aufgeforstet, die ein rasches Wachstum und hohe Erträge versprachen. "Das Waldbild wandelte sich. Wo noch unsere Großeltern und Eltern unter Eichen und Buchen wanderten, dehnten sich nachher Rottannenwälder aus», schrieb der kürzlich in hohem Alter verstorbene K. A. Meyer ${ }^{19}$.

Abschließend einige Detailbelege zur Illustration: 1713 enthielt das "Buchholz» ob Grafenried meistenteils erwachsene Eichen.

1752 wurden Eichen, Buchen, Tannen und Dählen genannt.

1776 war es ein Buchenwald.

Vermutlich ist das Buchholz nach 1800 nach einem Weideabtauschvertrag mit dem Staat Bern in Privatbesitz übergeführt und gerodet worden20.

In den Wäldern bei Laupen war um 1865 noch ein Eichenbestand von 5,31 ha vorhanden, der 1885 auf 0,8 ha zusammengeschrumpft war'1.

Nachwirkungen jener einseitigen Umstellungen, die Meyer als Zeit der "Fichtenmanie»22 bezeichnet, sind noch heute erkennbar. So wurde 1968 in einem forstlichen Gutachten der Zustand der Wälder Vorder- und Hinter-Eichsberg (!) folgendermaßen charakterisiert: "Biologische Forderungen scheinen im Privatwald Grafenried keine Gültigkeit zu haben. Im Gebiet des Laubmischwaldes werden die gesellschaftseinheimischen Arten durch Nadelhölzer ersetzt mit einseitiger Bevorzugung der Fichten'23.»

In Grafenried haben die Güterzusammenlegung (Abschluß 1935) und die Waldzusammenlegung (Abschluß 1954) die formalen Úberreste der Dreizelgenordnung zum Verschwinden gebracht, verbesserte Produktionsbedingungen geschaffen und damit die moderne Kulturlandschaft entscheidend geprägt. Der Vergleich von TA 142 (1880) und LK 1147 (1954) zeigt in der Verteilung von Wald und Feld nur unwesentliche Veränderungen. Die Produktions- und Betriebsstatistik dagegen vermittelt ein Bild voller Dynamik (Fig. 3). Wir greifen den Getreidebau stellvertretend für den Ackerbau 
heraus. Er ist statistisch von 1885 an erfaßbar und zeigt bis zum Ersten Weltkrieg ein kontinuierliches Absinken der Anbaufläche, bedingt durch die infolge billiger Getreideimporte (Anschluß an den Weltmarkt) erzwungene Verstärkung der Milchproduktion ${ }^{24}$. Der Erste Weltkrieg zeichnet sich in der Kurve der Anbaufläche kaum ab, dagegen folgt in den vierziger Jahren ein kriegsbedingter Aufschwung, dem nach einem neuerlichen Rückgang seit Mitte der fünfziger Jahre ein anhaltender Aufstieg folgt. Dies ist das Resultat der zunehmenden Mechanisierung und Rationalisierung, wie sie sich aus den in Figur 3 angeführten Zahlen ablesen läßt. Im Gegensatz zum Ackerbau blieb die Milchproduktion weitgehend konstant. Diese Entwicklung ist für das ganze tiefere Mittelland charakteristisch (siehe auch Dotzigen).

Die Betriebsbesichtigung bei Herrn Danuser zeigte uns einen modern geführten Mähweidebetrieb (d. h. für das Vieh täglich eine Stallfütterung und ein Weidgang), dessen 24 ha Kulturland zu $60 \%$ aus offenem Ackerland besteht. Zwei Drittel des Ackerlandes dienen dem Getreidebau, ein Drittel dem Hackbau. Die Rauhfutterfläche $(40 \%$ des Kulturlandes) besteht zu zwei Dritteln aus Klee und zu einem Drittel aus Naturwiese. Naturwiese und die Hälfte der Kleegrasfläche werden geweidet. Der Fruchtwechsel spielt sich nach zwei verschiedenen Rhythmen ab:

\section{Variante A}

1. Umbruch von Klee zu Weizen

2. Hackfrüchte (Kartoffeln, Gemüse)

3. Weizen

4. Hackfrüchte (u. a. Erbsen)

5. Weizen oder Futtergetreide (Hafer, Gerste)

6. Kleewiese

7. Kleewiese

\section{Variante B}

1. Umbruch von Klee zu Hackfrucht (u. a. Bohnen)

2. Weizen

3. Roggen

4. Hackfrüchte (u. a. Kartoffeln)

5. Weizen mit Kleinsaat

6. Kleewiese

7. Kleewiese

Das Siedlungsbild spiegelt die veränderten Produktionsverhältnisse noch kaum. Anläßlich der Güterzusammenlegung der frühen dreißiger Jahre erfolgten noch keine Aussiedlungen. Der leichte Rückgang der in der Landwirtschaft Tätigen bei konstanter Einwohnerzahl (Fig. 2) bewirkt eine $\mathrm{Zu}$ nahme der Bautätigkeit. So ist in den letzten Jahren am Ostrand des Dorfes ein reines Wohnquartier entstanden, und weitere Wohnbauten sind am westlichen Dorfrand vorgesehen.
Nach der üblichen Gliederung der Gemeinden in verschiedene Typen ${ }^{25}$ gehört Grafenried zum Typus $\mathrm{K}$ mit der Bezeichnung «ländliche Gemeinde mit gemischt nichtlandwirtschaftlicher Bevölkerung». Zweifellos hat der städtische Sog Grafenried noch nicht erreicht. Da jedoch 1960 von den 290 in der Gemeinde wohnhaften Berufstätigen deren 126 wegpendelten, sind stärkere arbeitsmarktliche Beziehungen nach außen vorhanden, die sich in erster Linie nach Bern (32 Wegpendler), Fraubrunnen (30) und nach Jegenstorf (17) richten²6. Die Landwirtschaft schließlich vermittelte den Eindruck, daß ihre Umstrukturierung in Richtung einer noch rationelleren und stärker mechanisierten Bewirtschaftungsweise weitergehen wird.

\section{Heimiswil - Übergangszone vom tieferen zum höheren Mittelland}

Naturlandschaft, Geschichte, Landschaftswandel und Probleme der modernen Kulturlandschaft sind in der Literatur bereits dargestellt worden ${ }^{27}$.

Als Grundlage für das Herausarbeiten instruktiver Einzelheiten zum Problem des Landschaftswandels dient für alle Beispiele die Arbeit von A. Leibundgut-Mosimann über "Heimiswiler Bauern und ihre Höfe» 28 .

Zunächst ein Beispiel für den Rodungsvorgang29. Der hinterste Teil des Heimiswiltales im Bereich der heutigen Höfe Hinter-Dreien, Dreienberg und Dreienbergli war zu Beginn des 16. Jahrhunderts noch Wald. In einem Lehensvertrag von 1518 wurde dieser Wald wie folgt umschrieben:

"So da stoßet an Lüdis zu Garnöül gut und ufher an die Straas da dannen ... zum Rodten Boum, da dannen hinüber an das Horn untzit an die Straas die gan Lucern gaht, da dannen über das Gut zu Heimelsmat ...» Dieser Dreien-Wald und der benachbarte, namengebende Hof (Vorder-Dreien) gehörten 1347 je zur Hälfte dem Kilchherrn Rudolf Pfründer, Pfarrer in Heimiswil, und dem Junker Johannes von Mattstetten. Im genannten Jahr vergabte Rudolf Pfründer seinen Besitz dem Niederspital in Burgdorf. 1466 konnte die Stadt auch die zweite Hälfte erwerben. 1518 verlieh sie «... dem Erbaren Meister Hansen Glaser und allen synen Erben einen unseren eignen Waldt, so man nent den Treygen, gelegen in der Kilchöri Heimiswyl». Glaser und seine Nachkommen gingen mit dem Dreienwald sehr eigenmächtig um. Der Lehensherr, das Oberspital zu Burgdorf, sah sich deshalb 1627 veranlaßt, das Lehen mit folgender Begründung wieder an sich zu ziehen: " ... welcher wald aber sythero ußgerütet zuo fruchtbaren guoteren, acher, maten undt weyden gemacht undt darneben durch die besitzer derselben dergestalt so villfaltig wider Lechens 


\section{Fig. 5 Ausgewählte Wirtschaftsdaten III \\ Trachselwald \\ Schangnau}

a) Landwirtschaftliche Produktion
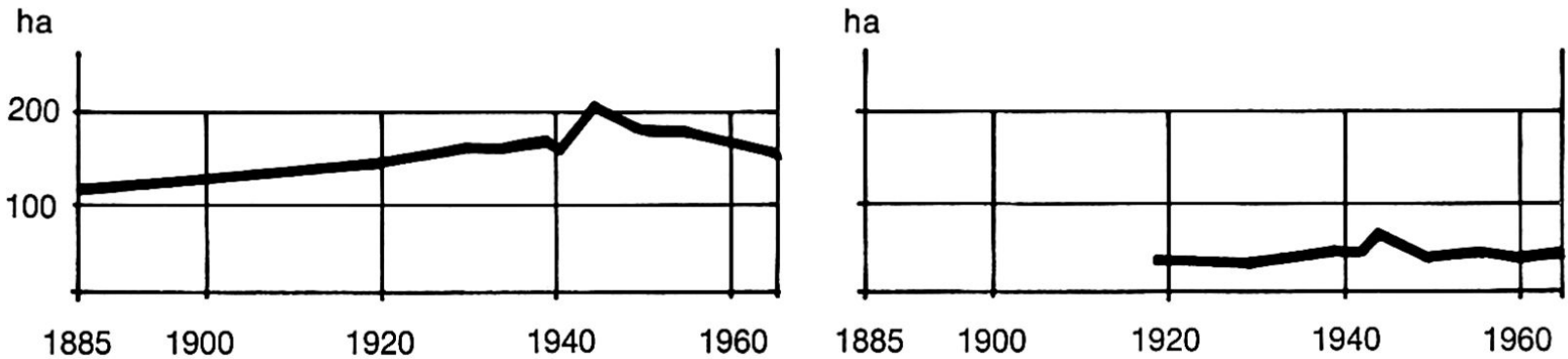

Getreidebau
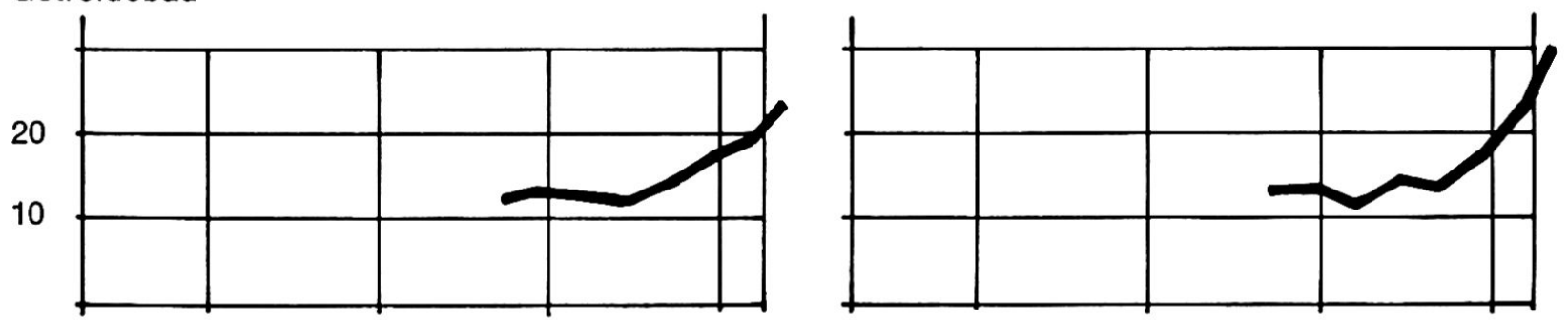

Milchproduktion in $1000 \mathrm{q}$
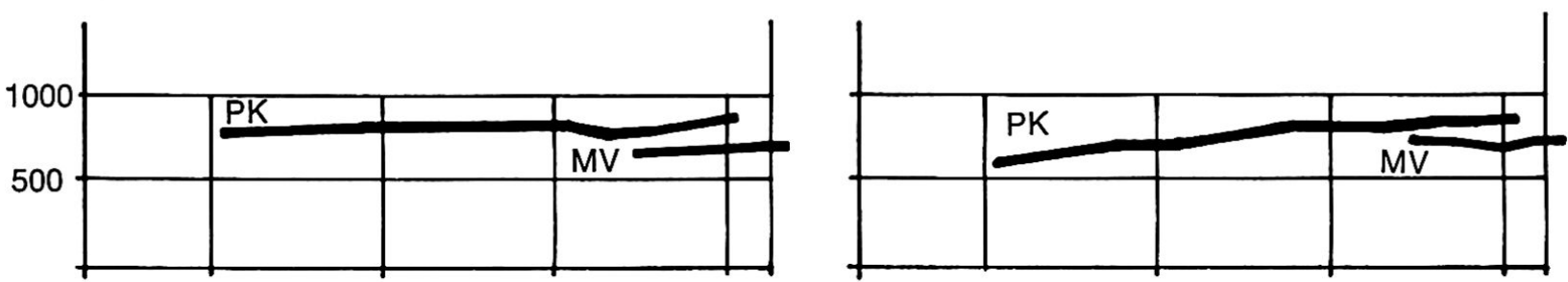

Milchkühe

b) Struktur der Landwirtschaftsbetriebe
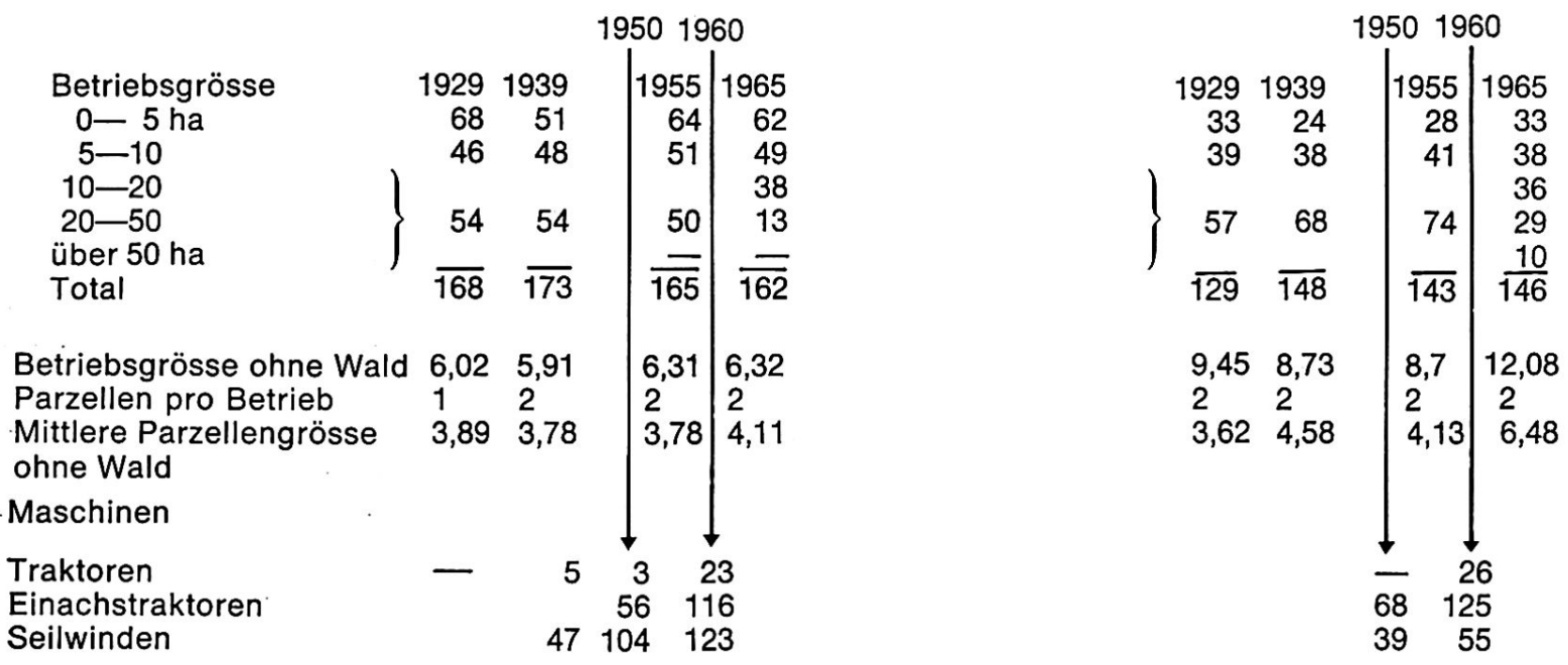

c) Industrie-, Gewerbe- und Dienstleistungsbetriebe (1. Zahl = Betriebe, 2. Zahl = Beschäftigte)

sekundärer Sektor tertiärer Sektor

46- $7926-52$

18- $26 \quad 17-27$

$\overline{64}-\overline{105} \overline{43}-\overline{79}$ 


\section{Fig. 4 Ausgewählte Wirtschaftsdaten II \\ Heimiswil \\ Rüegsau}
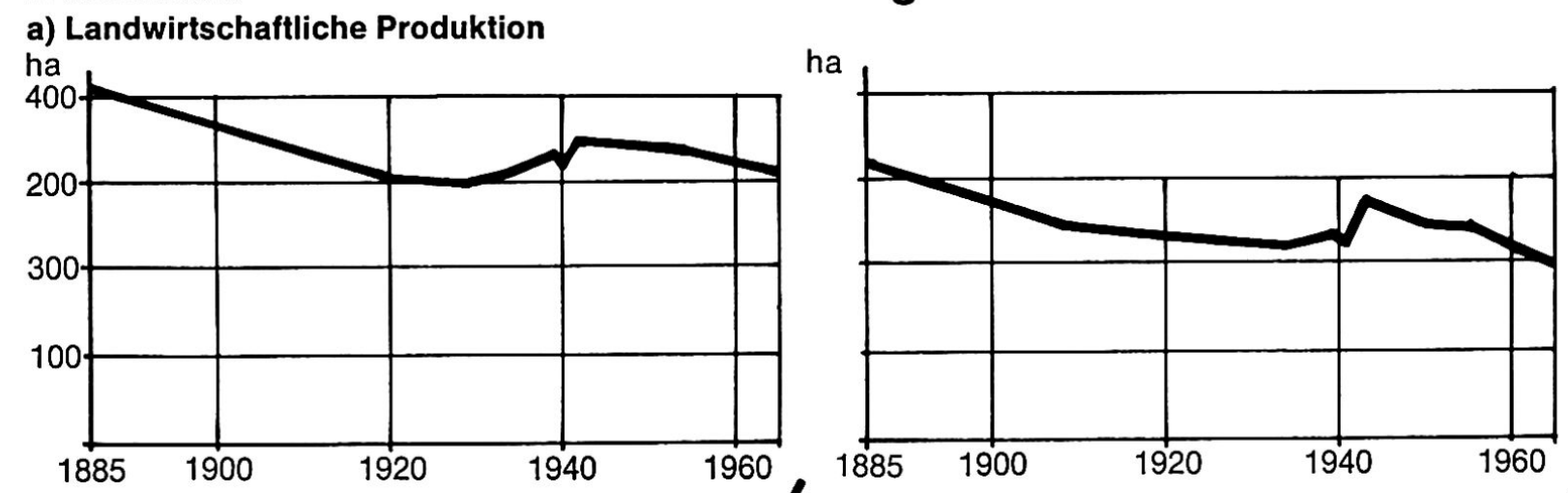

Getreidebau
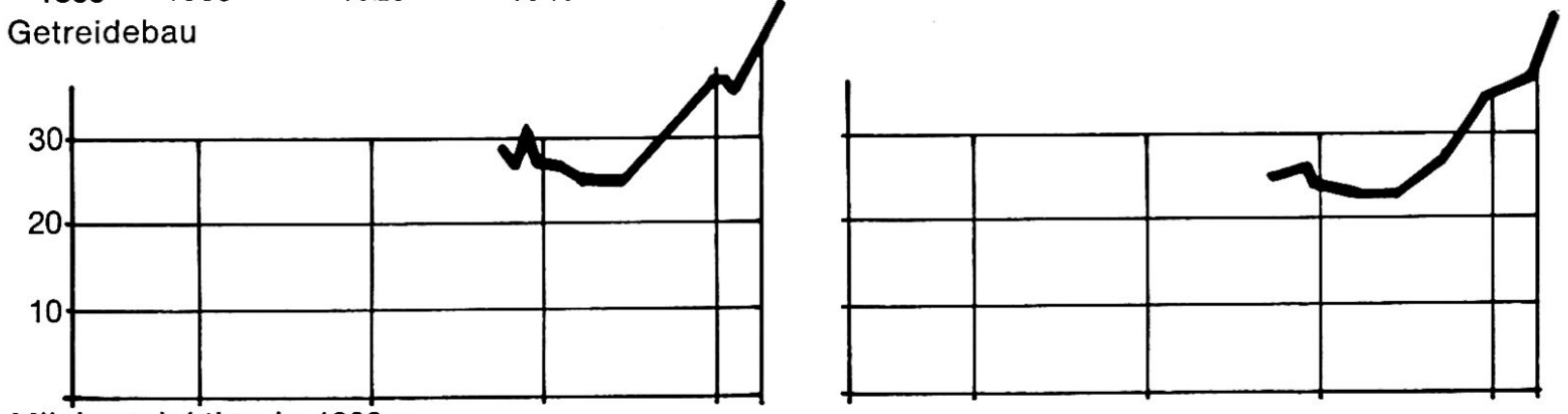

Milchproduktion in $1000 \mathrm{q}$
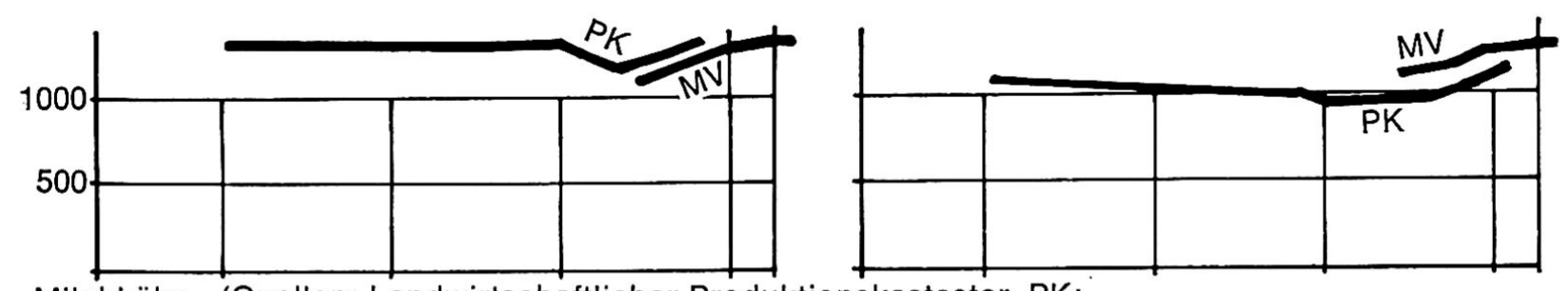

Milchkühe (Quellen: Landwirtschaftlicher Produktionskastaster, PK; Jahresberichte Milchverband Bern, MV)

b) Struktur d. Landwirtschaftsbetriebe 19501960

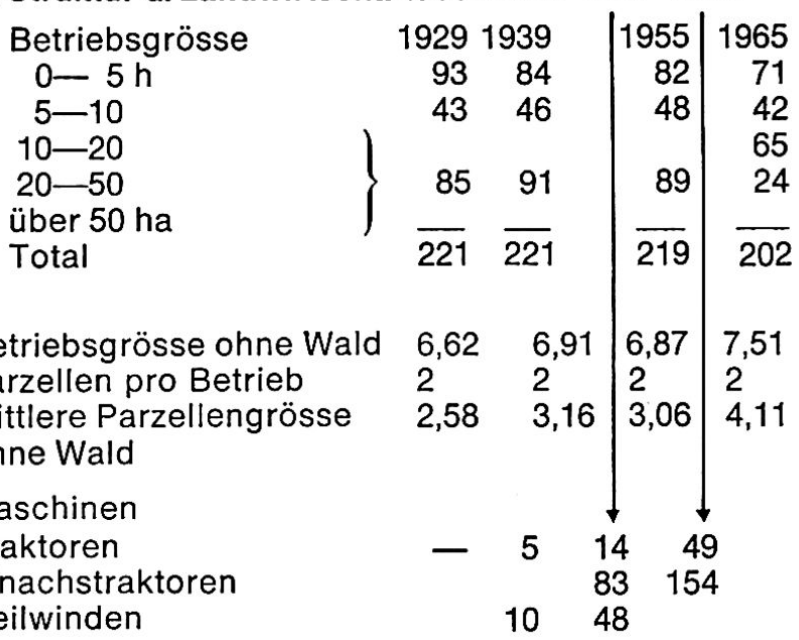

c) Industrie-, Gewerbe- und Dienstleistungsbetriebe

(1. Zahl = Betriebe, 2. Zahl = Beschäftigte)

19551965

50-103 $32-93$

$21-31 \quad 14-36$

$\overline{71-\overline{134}} \overline{46}-\overline{129}$

Total

Industriebetriebe mit $<20 \mathrm{~B}$.

20-99 Beschäftigte

$>100$ Beschäftigte

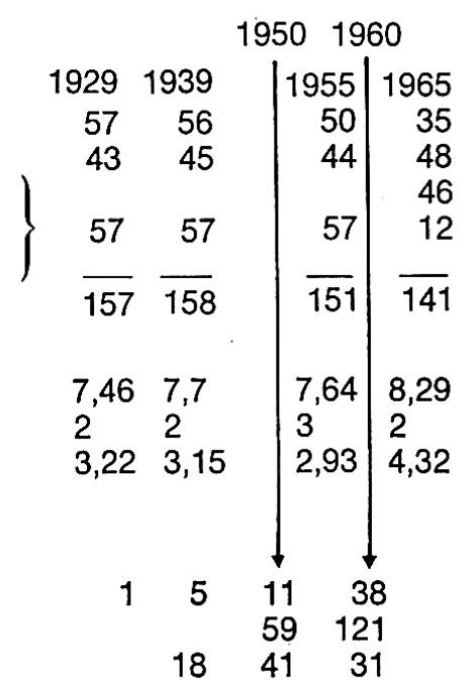

19551965

113-474 84-518

59-110 53-114

$\overline{172} \cdot \overline{584} \overline{137} \cdot \overline{632}$ 
recht und gewonheit, eigens gewalts zerstücklet, vertheylt, verkoufft und verenderet worden, das wir selbiges Gütli, die Treyen, mit Recht widrumb zuo unseren Handen bezogen ...» Nach dem Heimfall wurde das Lehen um den gleichen Zins von 14 Pfund an die Bauern Jost Widmer (Ober-Dreien), Hans Jau (Under-Dreien) und Hans Lüdi (Garnöu) ausgegeben.

Im folgenden wird der Vorgang einer Hofteilung skizziert, eines im Spätmittelalter und der frühen Neuzeit immer wieder praktizierten Verfahrens, um den verschiedenen Zweigen einer Familie einen eigenen Hof verschaffen zu können.

Der Hof Heimismatt (618 500/213 550/720 m), sonnseits gegenüber den Dreien-Höfen gelegen, ist erstmals 1340/41 erwähnt, als er als Kernstück des Kirchengutes Heimiswil an das Untere Spital in Burgdorf kam. Er umfaßte damals die beiden heutigen Höfe und das Areal des Hofes Gärstler. Der Stammhof wurde von 1340 bis 1508 von der Familie Meyer bebaut, deren erster bekannter Vertreter von 1340 bis 1377 als Walter von Heymoltsmatte bekannt war. 1508 erscheint in den Urkunden die Familie Großclaus. 1529 bestanden schon mehrere Häuser, Scheunen und Speicher. 1573 taucht ein Peter Aebi auf, der offenbar den zweiten Heimismatthof bewirtschaftete. Von 1588 bis 1895 blieb das ganze Lehen im Besitz dieser Familie, wobei 1688/89 der Hof Gärstler durch einen Seitenzweig dieser Familie errichtet wurde. Durch Einheirat änderten sich 1758 und 1785 auf zwei Höfen die Familiennamen. Handwechsel erfolgten erst 1895 und 1902 bei den beiden Heimismatthöfen ${ }^{30}$.

Eine dritte Betrachtung gilt der Dreizelgenwirtschaft. Während im würmeiszeitlich vergletscherten tieferen Mittelland im Bereich der großflächigen Siedlungsräume dörfliche Flurgenossenschaften entstanden, ermöglichten die Geländeformen im ehemals unvergletscherten höheren Mittelland (auBerhalb der Talterrassen) nur die Schaffung von Kleinsiedlungen, wie sie sich in jahrhundertelanger Rodungsarbeit in der Form der Einzelhofsiedlung ausgebildet haben. Die weitgehend zentrische Anordnung der Nutzungszonen mit abnehmender Bewirtschaftungsintensität nach außen (von Garten und Hofstatt zu Ackerland und danach zur Allmend $=$ Weide und Wald) war sowohl der Dorfsiedlung wie dem Einzelhof eigen. Der Einzelhofbauer konnte sich im Gegensatz zum Dorfbauern der Nutzungsfreiheit und des arrondierten Grundbesitzes erfreuen.

Als Zwischenstufe der Siedlung finden wir in Heimiswil auf der Egg - einer nach NW abfallenden Hochfläche zwischen Heimiswilgraben und dem Trockental Burgdorf-Wynigen, die nur während des würmeiszeitlichen Eishöchststandes vergletschert war - und auf dem Talboden des Heimiswilgrabens einige Weiler, in welchen ehemals auch nach dem Dreizelgensystem gewirtschaftet worden ist. Dies war der Fall in Guetisberg, Wil, Hueb, Dörfli (Ober-Heimiswil), Niederdorf, Chipf und Bußwil ${ }^{31}$.

Über die Anfänge der Dreizelgenwirtschaft stellt F. Häusler ${ }^{32}$ fest, «daß man diese heute nicht mehr als ursprünglich germanische Flurordnung, vielmehr als das Ergebnis eines jahrhundertelangen Strebens nach verbesserter Bodennutzung betrachtet». Interessante Hinweise liefert nun das kiburgische Urbar von 1261/1263, das die Zinsen der in 9 Ämter oder Einnehmereien gegliederten kiburgischen Höfe aufführt ${ }^{33}$. Zum Amt Guetisberg gehörten 16 Höfe im Oberaargau, davon vier auf dem Guetisberg selbst. Die Besitzer dieser 16 Höfe und jene weiterer Schupposen hatten den Kiburgern jährlich rund 32 Mütt Dinkel, Roggen und Haber, 37 Schweine, 108 Laffli und Schüfeli, 80 Lämmer, 54 Hühner, 480 Eier, 71/2 Schilling Bargeld, 135 Ellen Leinwand und 100 Tonschüsseln oder Chacheli abzuliefern. Die folgenden 140 Jahre brachten den Zerfall des kiburgischen Besitzes, und 1401 erwarb der Burgdorfer Conrad Stampf die Guetisberghöfe. 1419 wurde der Zins für die vier Höfe neu umschrieben. Er betrug von da an 331/3 Mütt Dinkel und Haber, 24 Hühner, 160 Eier und 2 Pfund Pfennige. Da die 4 Guetisberghöfe so viel Getreide abliefern konnten wie 150 Jahre vorher die 16 Höfe und 6 Schupposen des ganzen Amtes Guetisberg, taucht die Frage auf, ob sich von 1260 bis 1419 die Wirtschaftsweise verändert hat ${ }^{34}$. Wurde der Zins als Folge eines allgemein intensiveren Anbaues neu umschrieben oder wird die Umstellung von einer gemischten Wirtschaftsweise (Ackerbau und Viehzucht) zu einer im Dreizelgensystem betriebenen Getreidewirtschaft faßbar?

Die Gegenfrage nach dem Zeitpunkt der Auflösung der Dreizelgenordnung kann im Bereich der Weiler von Heimiswil eindeutiger beantwortet werden 35 . In den nach der Reformation aufgenommenen Urbarien wurden die Zelgen der Weiler-Flurgenossenschaft Guetisberg, Wil, Hueb, Niederdorf, Chipf und Bußwil erwähnt. Gegenüber der oben aufgestellten Liste fehlt die Gebursami von Ober-Heimiswil. Die Parzellen der drei dazu gehörenden Höfe wurden jedoch angegeben und befanden sich verteilt je am Schindlenberg (617 800/212 500), auf Schwändi (617 300/212 500) und im Längebächli (617 100/213 100). Offensichtlich ist in Ober-Heimiswil, in unmittelbarer Nähe der arrondierten und in selbständiger Wirtschaftsweise bebauten Berghöfe, bereits in vorreformatorischer Zeit das Prinzip der genossenschaftlich gebundenen Bewirtschaftung aufgehoben worden. Der Anstieg der Bevölkerung verlangte eine intensivere Bewirtschaftung, die nach einer frühen genossenschaftlichen Phase nur durch einen Übergang zu individueller Wirtschaftsweise zu erreichen war. 
In diese Intensivierungsbestrebungen hinein sind einmal die bereits erwähnten Rodungen in der Dreien zu stellen, ferner im Bereich der nicht arrondierten Höfe von Ober-Heimiswil private Güterzusammenlegungen zwischen 1580 und 1654, die mit der Verlegung der Stammhäuser aus unmittelbarer Nähe der Kirche nach dem Hofacker (616 450/212 $600)$ und dem Sonnberg (616 700/213 050) verbunden waren. Nach 1580 wurde mit der Verteilung der von der Bursami Oberheimiswil und den Einzelhöfen Dreien, Garnöu, Heimismatt und Rumstal genutzten Allmend begonnen. Zur Allmend wurde alles gezählt, was außerhalb der als Privateigentum eingezäunten Hofstätten, Matten, Äcker und Zelgen lag. Zur Sommerzeit diente sie dem Weidegang. In den höheren Lagen bestand die Allmend aus einem als Ußweide bezeichneten lichten Eichen-, Haselund Hainbuchenwald. Zu den erwähnten Höfen gehörten die ausgedehnten Weidwälder zwischen Cholgrueben (618 200/211 800) und Dreien, die noch heute die Namen "Weidwald» und «Hofacherweid" tragen, sowie die Ußweide zwischen Rüüglen (616 150/212 750) und Sunnberg. Die Verteilung der Allmenden ging schrittweise vor sich. Die dem einzelnen Bauern zugesprochenen Gebiete wurden in den dem Hof näher gelegenen Teilen gerodet und angebaut, während in den entfernteren Teilen der Weidgang allmählich unterblieb und sich der übernutzte und geschädigte Wald erholen konnte. Das letzte Teilstück der alten Allmend wurde 1875 von der Einwohnergemeinde Heimiswil, der Rechtsnachfolgerin der ehemaligen Allmendbesitzer von OberHeimiswil, in Privatbesitz verkauft. Damit hatte ein Vorgang den Abschluß gefunden, der im oberen Teil des Heimiswilgrabens aus den «alten» Einzelhöfen Rumstal, Heimismatt, Dreien, Garnöu und den ursprünglich drei Höfen der Gebursami OberHeimiswil durch Rodungen, Hof- und Allmendteilungen rund 40 größere und kleinere Heimwesen hatte entstehen lassen ${ }^{36}$.

Die Auflösung des Flurverbandes Ober-Heimiswil ist kein Sonderfall. Wie F. Häusler ${ }^{37}$ ausführt, ist der Zerfall der Weiler-Flurgenossenschaften im Emmental und das Umlegen des Landes zu mehr oder weniger arrondierten Höfen eine allgemeine Erscheinung des 16 . und 17. Jahrhunderts.

Fassen wir zusammen: Die von den GuetisbergHöfen zu entrichtenden Bodenzinsen stellen die Frage, ob die Einführung der Getreide- und damit der Dreizelgenwirtschaft erst nach 1260 erfolgt sei. Die Guetisberg benachbarte Weiler-Flurgenossenschaft von Oberheimiswil wurde bereits knapp 250 Jahre später aufgelöst. Wer liefert zu diesem Problem weitere Beobachtungen?

Die Probleme der modernen Kulturlandschaft von Heimiswil sind in dieser Zeitschrift 1965 dargestellt worden 38 . Nach Figur 2 war Heimiswil 1960 als bäuerliche Gemeinde mit gewerblichem Charak- ter (Typ M) anzusprechen. Diese bodenständig wirkende Umschreibung ist trügerisch. 1960 fehlten für die 835 in der Gemeinde wohnhaften Erwerbstätigen 232 Arbeitsplätze innerhalb der Gemeinde (siehe Anhang). Dieser Mangel an Arbeitsplätzen und die schlechte Verkehrslage bewirken eine permanente Abwanderungstendenz, die von 1960 bis 1968 zu einem Rückgang der Bevölkerung um 42 Personen oder $2,1 \%$ geführt hat ${ }^{39}$. Da auch die landwirtschaftlichen Arbeitskräfte immer rarer werden, bildet sich der Getreidebau auf den nicht maschinell bearbeitbaren Molassesteilhängen zurück zugunsten der Milchproduktion (Fig. 4), wobei die Umstellung von Stall- zu Weidefütterung einen zusätzlichen Rückgang der Bewirtschaftungsintensität mit sich bringt.

\section{Heimisbach (Gemeinde Trachselwald) - Höheres Mittelland}

Der Untergrund der Naturlandschaft von Heimisbach ist durch die Molasse des Tortonien gebildet. Die Wechsellagerung von Mergel, Sandstein und Nagelfluh führte zur Gliederung der Talhänge durch ausgeprägte Denudationsterrassen ${ }^{40}$. Gegenüber der Landschaft von Heimiswil bis und mit dem tieferen Teil von Heimisbach fällt im Abschnitt Nülli (627 425/207 175/1039,1 m) - Sparrenegg (628 400/206 875/1055 m) die wesentlich reichere Durchtalung auf: der Übergang von dem rißeiszeitlich vergletscherten zum unvergletscherten Gebiet des zentralen Napfberglandes wird erkennbar ${ }^{11}$.

Für die siedlungsgeschichtlichen Angaben sei auf die neue Arbeit von F. Häusler hingewiesen42. Das Emmental oberhalb von Burgdarf ist frühmittelalterliches Kolonialland, über dessen früheste $\mathrm{Be}$ siedlung keine Nachrichten überliefert sind. Die Urkunden, die erst im 12. Jahrhundert reichhaltigere Angaben liefern, lassen folgende Besiedlungsphasen vermuten:

a) Als Ausgangslage kann die teilweise auf gallorömische Wurzeln zurückführbare Besiedlung der Orte am Hangfuß zwischen höherem und tieferem Mittelland und im Aaretal gelten (z. B. Lyssach, Rüfenacht, Wichtrach). In dieser Zone der Dorfsiedlung finden sich im 12. Jahrhundert die kleinen Herrschaftsbereiche des zähringischen und später kiburgischen Ministerialadels.

b) In einer ersten Etappe erschienen (erst im 12. Jh. belegt) die großen Herrschaftsbereiche der Freiherren von Brandis, Sumiswald und Signau. Damit deutet sich wohl die Verteilung der Waldgebiete des Haupttales und der grö3eren Nebentäler (Zone des rißeiszeitlich überformten Gebie- 
tes) durch das Reichsoberhaupt an adelige Lehensträger $\mathrm{ab}$, die danach die Besiedlung dieser Täler in die Wege leiteten.

c) In der zweiten Etappe, im Anfang des 12. Jahrhunderts, wurde die Besiedlung des eigentlichen Oberemmentals (Ilfistal und Oberlauf der Emme) durch den Adel mittels Klostergründungen in Trub und Röthenbach gefördert.

Um 1300 scheint die Besiedlung des Emmentals bis auf zwei Zonen abgeschlossen zu sein: der überschwemmungsgefährdete Talboden der Emme und der breiteren Seitentäler sowie die Wälder über $1000 \mathrm{~m}$ (grob umrissen: das riß- und würmeiszeitlich unvergletscherte Gebiet) bleiben vorerst unbesiedelt.

In Heimisbach wurden 1278 das Gut Tal (625 850/ 207 600/745 m) und die Mühle «zem Brunne»sowie 1293 der Hof Eigen erwähnt.

Als Bestandteil des Gerichtes Trachselwald kam Heimisbach aus der Hand des Freiherrn Burkhard von Sumiswald im Jahre 1408 an Bern. Die Burg Trachselwald wurde zum Sitz des bernischen Landvogtes ${ }^{43}$.

Wenden wir uns nun der Betrachtung der Fluren zu. Die 1278 erwähnte Mühle im Heimisbach gestattet den Schluß, daß die tiefer gelegenen Höfe Ackerbau betrieben. Schon viel früher, nämlich im Stiftungsbrief der Deutschherrenkommende Sumiswald von 1225, sind die Alpen Arni und Nidungen erwähnt, womit für den bis über $1200 \mathrm{~m}$ hinaufreichenden Höhenzug zwischen Hornbach und Kurzeneigraben die Alpwirtschaft bezeugt ist ${ }^{44}$.

F. Häusler vermutet, daß die um 1300 festgestellte Erschließung des Emmentals im 14. und 15. Jahrhundert kaum oder nur wenig verändert worden ist ${ }^{45}$. Gegen Ende des 15. Jahrhunderts setzte eine rasche Zunahme der Bevölkerung ein, was sich urkundenmäßig durch die Bewilligung zahlreicher Rodungen niedergeschlagen hat ${ }^{46}$.

Für die Rückbildung der Hochwälder liefert Heimisbach eindrückliche Beispiele: 1339 erwarb Ulrich von Signau den Hochwald Sparrenegg aus der Hand des Junkers Dietrich von Rüti47. Im Jahre 1480 bestätigte der Landvogt Gilgian Schöni von Trachselwald eine schon früher erfolgte Verleihung eines Stückes Hochwald auf der Sparrenegg. Auf der gerodeten Fläche wurde Alpwirtschaft betrieben, der Bodenzins dafür betrug ein Mäß fetten Käses ${ }^{48}$. Bereits vor 1498 wurde auch auf der Lüdernalp geweidet ${ }^{49} .1513$ und 1549 wurden dem Besitzer des Bachgutes zuhinterst im Heimisbach (627 000/208 000/800 m) neben der bereits bestehenden Rodung drei weitere bewilligt, die 1569 als Habchschwendi, Große Schwendi und Surgraben bezeichnet wurden 48 .

Der Landhunger der nachreformatorischen Zeit ist vom Schreiber des Sumiswalder Urbars 1572 sehr plastisch umschrieben worden: "Ist geschehen zuo guotem teil von menschen gedechtnus ob und von den 40 Jahren har, das ein erliche herschaft der statt und landschafft Bernn, mit den iren sich frömbder unnotwendiger kriegen abgethan und entschlagen, dadurch uss gottes gütte ir land und erdttrich sich mit arbeit, rütten und buwen ufgethan und an mannschafft zugenomen, das sich nit eim jettlichen ein eigener hoff und guott mogen ziechen. Dann das sich ir vil erlicher redlicher gsellen lanndt kind von den Patriotis erboren und erzogen, mit sölichen hütten und schachenhüslinen (den altten vättern glich) vermügen umbsechen, unnd mit wyb unnd kinden, durch ir handarbeit, als taglöner müssen erneren. Wie das allenthalben in miner $\mathrm{g}$. Herrn von Bern lannden an wassern, in schachen, uf allmenden, zu holz und velde, unden und oben bis an die ussersten grennze brüchlich unnd zesechen.

Also ist es ouch nit minder zuogangen in der Herschaft Sumiswald, ... davor nachher schier ein jettlicher, mangelhaffter (d. h. jeder, dem es an Land mangelte) sich ouch ingeseztt unnd hüser buwen, hofstaten, bünden unnd gertten ingeschlagen, schier ungefragt und ane rechnung. Von voran zu Fürtten und Grünen, demnach der Grünen, dem Hornbach unnd Wasen nach in (besunder zu Grünen, mit hanntwerkern und taglönern schier zu einer vorstatt geratten) bis zehinderst in allen vierteilen, winkeln unnd ortten wo ein komlicher plaz mogen ... funden und eräfert (verbessert) werden . . .50.» Die nachteiligen Folgen der übermäßigen Entwaldung blieben auch den Zeitgenossen nicht verborgen. So bemerkte ein Landmann vom Kurzenberg im Jahre 1599: "Es syenndt die waldwasser jetziger zyt nit in iren alten rünsen zeerhalten, wie vor dryßig oder viertzig jaren, uss ursachen, das die weld und hölzer dermaßen uffgethan und ußgrüttet, das das wasser mit yll zu allen orten in sölche greben und bäch zusammen fließe, welches sich aber ein zyt lang in den thannen und studen gesumpt...51.»

Die Vergrößerung der Kulturfläche führte zu weiteren Differenzierungen. So wurden zunächst größere Höfe geteilt. Als Beispiel aus dem Heimisbach sei die Dreiergruppe «Unter Schwarzenegg» $(780 \mathrm{~m})$, "Mittlere Schwarzenegg» (625 250/206 300/851 m) und "Hinter Schwarzenegg" (856 m) erwähnt52. Ferner können entsprechend der Höhenlage verschiedene Bewirtschaftungsformen unterschieden werden. Als tiefstgelegenes Beispiel dient der Hof "Zur Mühle» im Heimisbach. Er wurde $1569 \mathrm{mit}$ folgenden Worten umschrieben: «Ist ein ingefangen guetli (Einzelhof), hus, hof, matten, weid und holtz, alls in eim infang, in einer züni (Zaun); by 6 meder maten; der Lengacher, der Krumacher und Spicheracher, by 8 jucharten; das ander ist weid, holtz und schachen ${ }^{53 . » ~ O f f e n b a r ~ k o m m t ~ h i e r ~ d e m ~ G e t r e i d e-~}$ 
bau eine wichtige Rolle zu, werden doch drei Äcker namentlich erwähnt. Die Vermutung ist sicherlich gestattet, daß der Bewirtschafter das Dreizelgensystem praktizierte und es sich damit um einen sogenannten Zeitzelgenhof handelte, dessen Ackerflur nach den starren Regeln der Dreizelgenwirtschaft bebaut wurde 54 . In der Umschreibung des Gutes Twiri aus dem gleichen Jahr wird der Getreidebau auf dem auf der Nordseite des Heimisbaches liegenden Hofes (626 300/208 300/890 m) weniger betont: "Ist darin hus, hof, spicher, by 12 meder maten und acher, das ander holtz, veld und weid55.» Die Angaben lassen schließen, daß wir es hier mit einem Agertenhof zu tun haben, bei welchem das Ackerland nach sechs oder mehr Jahren zu Wiesund Weideland wurde und durch eine neue Fläche Umbruch ersetzt wurde. Auf diese Weise verschob sich das Ackerland im Laufe der Jahre nach freiem Ermessen des Bewirtschafters über das ganze Hofgebiet, wobei der sich nach und nach verlagernde und turnusmäßig alles Kulturland erfassende Umbruch einen verbesserten Futterbau bewirkte56. Im dritten Beispiel aus dem gleichen Urbar von 1569 wird bei der Umschreibung des am südlichen Rand des Heimisbach liegenden Gutes St. Oswald (627 450/205 250/990 m) kein Ackerland mehr erwähnt, womit der Anschluß an die Stufe der Alpwirtschaft hergestellt sein dürfte: "Ist hus, hof, matten, holtz, veld, weiden, alls in einm zun ${ }^{55}$.»

Die steigende Volkszahl zwang die Emmentaler nicht nur zur Vergrößerung der Kulturfläche und zur Erhöhung der Anzahl Höfe; sie führte auch zu einer intensiveren Nutzung der Fluren. Im 16. Jahrhundert lag das Schwergewicht auf der Ausdehnung des Ackerbaues. Damit wurde die extensive Weidewirtschaft zurückgedrängt und "laufend verwandelten sich Alphütten in dauernd bewohnte Bauernhöfe»57. Wir erwähnen hier den Hof Zueguet (628 025/207 150/1038 m), wo 1868 Simon Gfeller geboren wurde. Die Rodung Zueguet ist 1569 bewilligt worden. Zunächst entstand eine Alp, auf der 18 Rinder gesömmert werden konnten. 1626 wurde die Alp im Urbar von Trachselwald als Hof Zueguet aufgeführt 58 .

Im Übergang zum 18. Jahrhundert setzte eine weitere Intensivierung der Bewirtschaftung ein, die von der Selbstversorgung weg zur Marktwirtschaft führen sollte: Im Oberemmental begann man den Getreidebau zugunsten der nach dem System der Ägertenwirtschaft angebauten Kleewiesen einzuschränken. Nach dem Bericht von Pfarrer Schweizer aus Trub mußten die Müller des Emmentals um 1764 wöchentlich ganze Fuder Getreide in Burgdorf holen, damit der Bedarf an Brot gedeckt werden konnte58. Mit der Zunahme des Futterbaues stieg die Milchproduktion und damit konnte mehr Käse hergestellt werden, der als Exportgut vom Ende des 17. Jahrhunderts an immer wichtiger wurde. Parallel dazu entwickelte sich das Küherwesen 59 .

Im tieferen Mittelland blieb die Dreizelgenwirtschaft noch bestehen. Sie verschwand erst, als das; von Gotthelf in der "Käserei in der Vehfreude» geschilderte "Käsefieber» im zweiten Viertel des 19. Jahrhunderts auch die Dörfer erreichte. Damit verloren die Küher die Futterbasis für ihre Herden, und die Käsefabrikation verlagerte sich definitiv in die Täler und Ebenen hinunter60.

Welche Struktur und welche Umwandlungstendenzen zeigen sich in der jetzigen Kulturlandschaft von Heimisbach? Im Unterschied zum Trub oder etwa zum angrenzenden Goolgraben 61 zeigt der Kartenvergleich zwischen 1885 (TA Blatt 196) und 1955 (LK Blatt 1148), daß die Waldfläche sich nur unwesentlich verändert hat.

Die Kurve des Getreidebaues (Fig. 5) belegt, daß im Heimisbach von 1885 - weiter zurück fehlen genaue Angaben - bis in die dreißiger Jahre hinein eine kontinuierliche Intensivierung der Betriebe erfolgt ist. Diese Erscheinung gilt auch für das übrige Amt Trachselwald, da die Getreideanbaufläche des Bezirks von 1917 bis 1929 um 27 ha auf 2327 ha gestiegen ist.

Nach dem Uberblick das Detail: Bis um 1920 war der Hof Nülli (627 525/207 075/1010 m) ein Weidebetrieb des Gehöftes Mistebüel (626 500/206 900/830 m). Heute wird das Kulturland des Hofes Nülli wie folgt genützt: 0,9 ha Getreide, 0,7 ha Kartoffeln, 3,8 ha Wiesland, 0,3 ha Weide. Der Fruchtwechsel entspricht dem Ägertenprinzip: erstes Jahr Umbruch von Wiesland, Kartoffelanbau, zweites Jahr Wintergetreide mit Kleesaat, drittes bis sechstes Jahr Kunstwiese. Da die Entfernung zur Talkäserei zu groß ist, wird die Milch zum Mästen von Kälbern verwendet.

Nachdem der Zweite Weltkrieg den Anbau von Getreide nochmals gefördert hatte, hat sich seither die wirtschaftliche Lage der Gemeinde weniger erfreulich entwickelt (Fig. 5). Zunächst ist die Struktur der Bauernbetriebe seit 1929 praktisch gleich geblieben. Die übrigen Wirtschaftszweige haben sich sogar zurückgebildet. So ist die Zahl der Gewerbebetriebe im Jahrzehnt 1955-1965 um ein Drittel und die Zahl der Arbeitsplätze um ein Fünftel gesunken. In der Arbeitsplatzbilanz fehlen innerhalb der Gemeinde 57 Arbeitsstellen, so daß der von 1960 bis 1968 festgestellte leichte Bevölkerungsrückgang ( $1,6 \%$ oder 20 Personen) oder mindestens eine Stagnation anhalten dürfte (siehe Anhang).

Wie die Bevölkerungspyramide zeigt (Fig. 6), fehlen in der Bevölkerung von Heimisbach vor allem die im besten Erwerbsalter stehenden mittleren Jahrgänge und deren Kinder. Nach den Aussagen des Gemeindepräsidenten, Herrn Paul Gfeller vom Hinder Liechtguet, ist vor allem der Mangel an jungen Bäuerinnen sehr kraß, so daß die Weiterfüh- 
Fig. 6

Gemeinde Trachselwald — Bevölkerungspyramide 1960

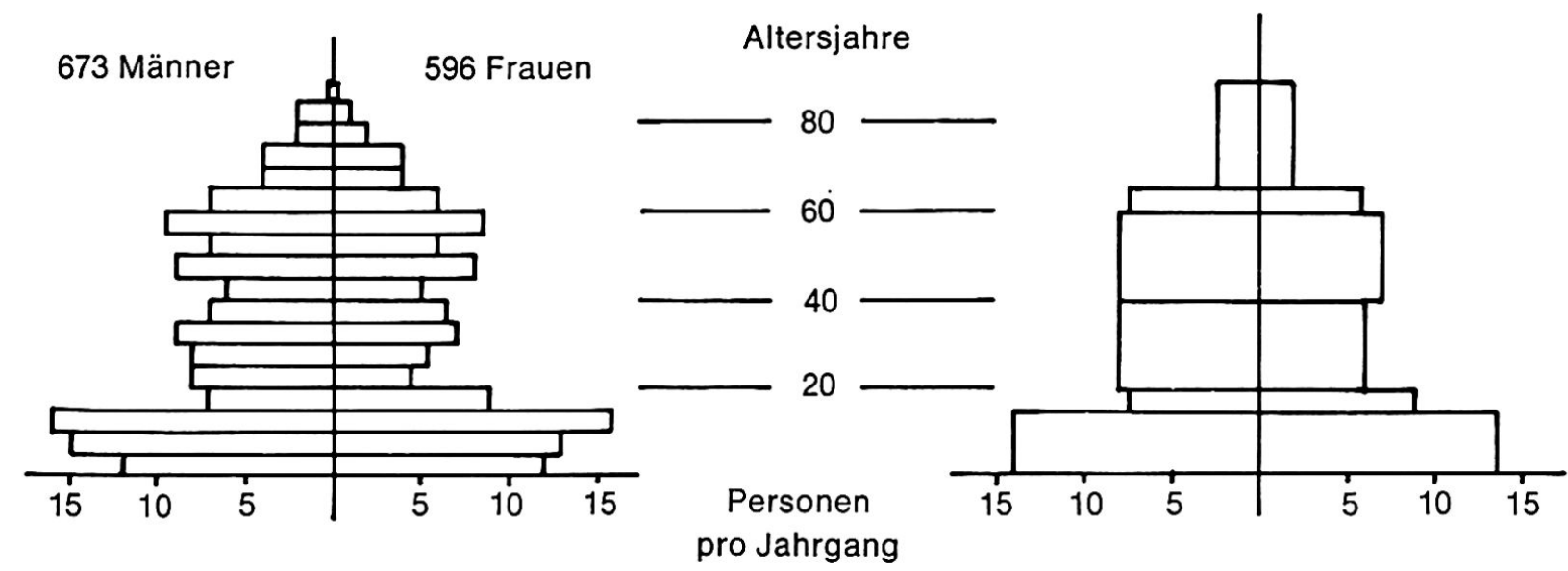

Figur 6. Gemeinde Trachselwald. Bevölkerungspyramide 1960. Die Pyramide rechts ist nach den vom Eidgenössischen Statistischen Amt publizierten Werten gezeichnet (VZ 1960, Q 364). Sie zeigt, daß die Altersstufen zwischen 15 und 40 Jahren verhältnismäßig schwach vertreten sind (Abwanderung). Die große Zahl der Kinder unter 15 Jahren deutet auf eine gesunde Basis. - Die Pyramide links ist nach Fünfjahresstufen aufgebaut (unpublizierte Maschinentabellen des Eidg. Stat. Amtes). Sie gestattet,

rung vieler Betriebe fraglich geworden ist ${ }^{62}$. Der Verlust an Arbeitskräften konnte nur teilweise durch die Anschaffung von Maschinen ausgeglichen werden. So ist denn der Getreidebau seit dem Zweiten Weltkrieg dauernd zurückgegangen. Viele Betriebe dehnen zurzeit die. Weidefläche aus, da der Úbergang zur gesteigerten Milchproduktion der einzige Ausweg ist, um trotz mangelnder Arbeitskräfte den Hof weiterhin bewirtschaften zu können. Bei mangelhafter Pflege der Weidefläche geht die Umwandlung von Weide zu Wald rasch vor sich. Daher ist die oben für den Zeitraum 1885-1955 festgestellte Stabilität in der Verteilung von Wald und Kulturland für die Zukunft nicht mehr gesichert.

\section{Die Schachensiedlungen}

Für die Naturlandschaft und die allgemeine Entwicklung der Schachensiedlungen sei auf die Arbeit von E. Gerber über «Die Flußauen in der schwei- den demographischen Aufbau wesentlich präziser zu interpretieren. Die in der Pyramide rechts festgestellte "gesunde» Basis ist in Wirklichkeit nicht mehr vorhanden. Im Gegenteil, die Kinderzahlen nehmen stark ab, da die eigentliche Elterngeneration zahlenmäßig geschwächt ist. - Die beiden Darstellungsweisen zeigen, daß die übliche Zahlenpublikation in zusammenfassenden Altersgruppen sehr summarisch ist und daß die durchgehende Angabe der Fünfjahresgruppen wünschbar wäre.

zerischen Kulturlandschaft» 63 hingewiesen. Die eingehende Darstellung der Besiedlung und weitere Literaturangaben finden sich in der schon oft zitierten Arbeit von F. Häusler64.

Figur 7. Siedlungsentwicklung in Rüegsauschachen. 1. Siedlungsausdehnung im Anfang des 19. Jahrhunderts; 2. Siedlungsausdehnung 1871; 3. Siedlungsausdehnung 1965; 4. Hangfuß; 5. Wichtigste Straßenzüge 1965; 6. Emmeufer im Anfang des 19. Jahrhunderts; 7. Höfe außerhalb des Dorfes Rüegsau, die bis 1658 Weidrechte im Rüegsauschachen besaßen; 8. Holzbrücke I von 1764 bis 1837 ; 9. Holzbrücke II von 1839 bis 1955 , ersetzt durch Betonbrücke 1956; 10. Neuer Standort von Holzbrücke II ab 1957

Quellen: Übersichtsplan Blatt 145 (1965); TA 145 (1871): "Plan von einem Teil der Kirchgemeinden Lützelflüh und Rüexau» (undatiert, Anfang 19.Jh.); Würgler 1965, 164 


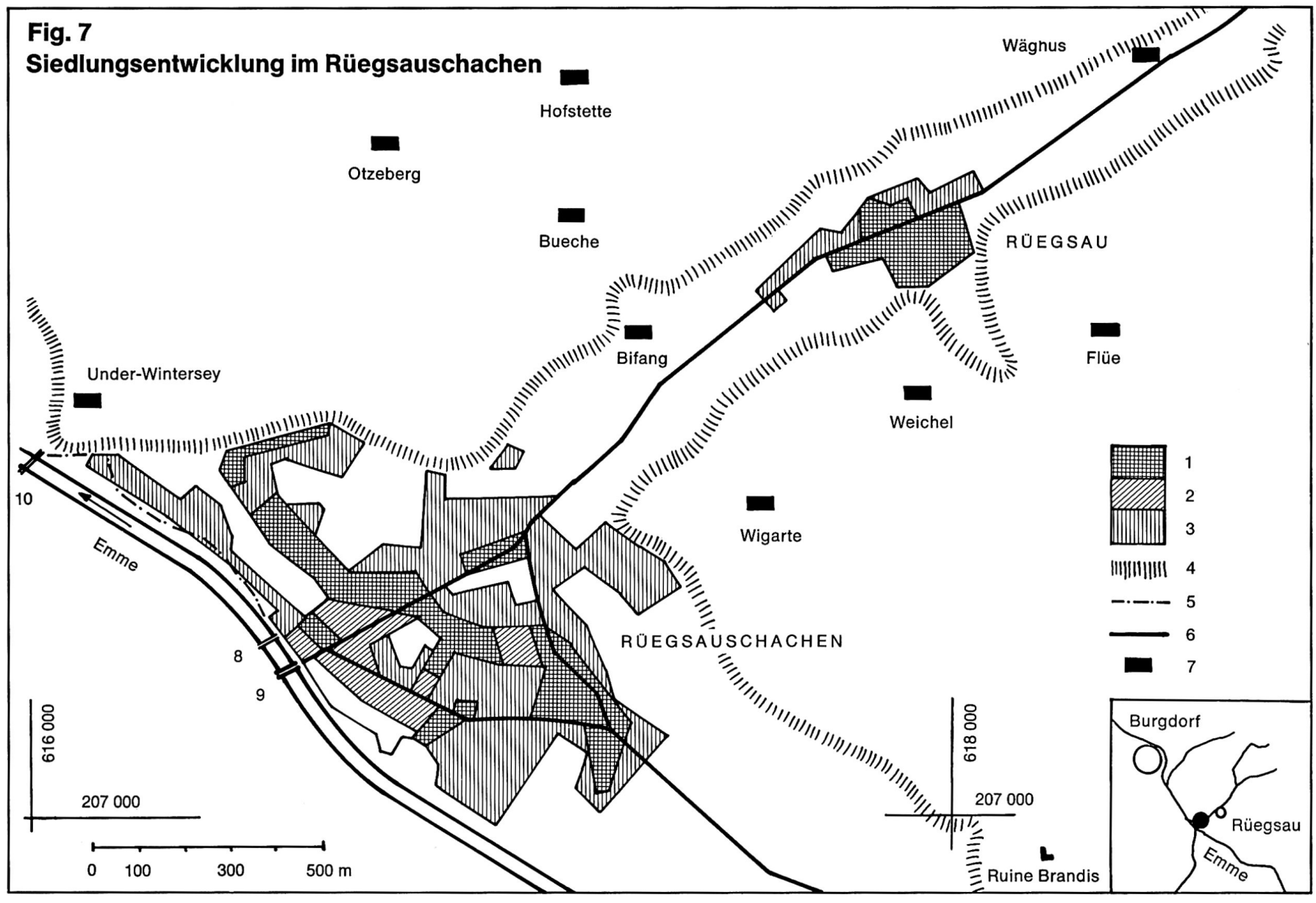


Wie im Abschnitt über Heimisbach dargestellt worden ist, bestanden im 15. Jahrhundert die Rodungsreserven der Hochwälder und der auf der häufig überschwemmten Talsohle liegenden Schachenwälder. In den Seitentälern der Ilfis und der Emme setzte die durch die steigende Bevölkerungszahl angetriebene Besiedlung der Schachen bereits im 15.Jahrhundert ein ${ }^{65}$, an den Hauptflüssen dagegen erst zu Beginn des 16. Jahrhunderts. So stand im Bomatschachen (in der Nähe der 1552 erbauten Zollbrücke) noch 1495 kein Haus66 und im Rüegsauschachen 1535 erst ein einziges, das einer ehemaligen Nonne des Klosters Rüegsau, der "Wallpurg tachs", und ihrem Manne gehörte ${ }^{67}$. Im 16. Jahrhundert war der heute intensiv genutzte und dicht besiedelte Schachen nur Randgebiet des menschlichen Wirtschaftsraumes. Der Verkehr führte nicht durch die Täler, sondern über die Höhen. Im Schachen ließ sich nur nieder, wer sonst nirgends unterschlüpfen konnte ${ }^{68}$. Überall, wo es ihnen zweckmäßig schien, legten Taglöhner und Handwerker in den Schachenwäldern größere Einschläge an, bauten ein kleines Haus und errichteten damit neue Dauersiedlungen. Damit wurde allmählich die Schutzwirkung des Uferwaldes reduziert, und zudem zahlten die Kleinsiedler keine Bodenzinse, da sie die Rodungen ohne Bewilligung durchgeführt hatten69. Die Obrigkeit begann daher im 16. Jahrhundert die Hoheit über die "Reisgründe» entlang der Emme zu beanspruchen und ihre Nutzung zu regeln. In der Landvogtei Trachselwald wurde die Inventarisierung der bisher illegalen Schachensiedlung 1568/69 vollzogen. So fanden die mit der Inspektion Beauftragten zum Beispiel im Gebiet von Grünenmatt und im Dürrgraben zehn Schachenhäuser und im Schachen von Rüederswil deren elf, von welchen bisher keines Zins entrichtet hatte 70 .

Durch die Landzuweisung im 16. Jahrhundert wurde der schützende Uferwald trotz aller Vorsorge verkleinert, wobei die armen Schachenleute aus materiellen Gründen die Schwellenpflicht nur ungenügend erfüllten. Die Entwicklung führte im 18. Jahrhundert dazu, daß die Schachenbewohner in Korporationen zusammengefaßt wurden, um deren Pflichten und Rechte genau regeln zu können ${ }^{71}$.

Am Beispiel des Schachens von Rüderswil kann aufgrund eines von $\mathrm{H}$. Michel umgezeichneten Schachenplanes von 1728 die damalige Landaufteilung gezeigt werden72. Vom Hangfuß der Niederterrassenschotter reicht die Zone der Parzellen, die sich im Sonderbesitz der Schachenleute befanden, etwa 80 bis $220 \mathrm{~m}$ weit in die Talsohle hinaus. Anschließend folgt ein dem Ufer entlang verlaufender 25-100 m breiter Streifen, der 1728 von der Obrigkeit als sogenanntes Trägereiland an die Korporation der Schachenbewohner verliehen und von diesen an ihre Mitglieder weitergegeben wurde. Da- nach folgte ein Hochwasserdamm und schließlich als letzter Streifen das 10-30 m breite Ufergehölz Die von 1884 an durchgeführte umfassende Emme korrektion ${ }^{73}$ reduzierte die Breite des Emmelaufe: im Abschnitt des Rüederswilschachens von $100 \mathrm{n}$ auf $30 \mathrm{~m}$ Breite. Damit konnte im letzten Jahrhun. dert die Fläche des Kulturlandes nochmals ausge. dehnt werden. Heute bietet die Schachenlandschaf im Bereich der Gemeinde Rüderswil den Eindruck einer intensiv landwirtschaftlich genutzten Talebene, die infolge ihrer schlechten Verkehrslag $\epsilon$ auch in der nächsten Zukunft nur wenige Verände. rungen erfahren wird.

Anders ist die Entwicklung im Rüegsauschachen verlaufen: Nachdem vor 1535 das erste Haus errich. tet worden war, siedelten sich bis 1576 weitere 12 Familien im Schachen an. Neben den kleinen Gschickli in der Größe von $1 / 2$ bis $11 \frac{1}{2}$ Jucharten wurden auch einige Gewerbe ${ }^{74}$ betrieben (Säge, Reibe, Walke, Badstube). Daneben besaßen seit alters Bauern von Rüegsau und der benachbarten Einzelhöfe das Recht, im Schachenwald das Vieh zu weiden und einzelne Einschläge während 6 Jahren privat zu bewirtschaften (Fig. 7). Wie im Laufe der Jahre die Zahl der Dauersiedler im Schachen zunahm, behielten die Bauern entgegen dem Rütirecht ihre Einschläge auch offen und verwandelten sie in permanente Fluren.

1587 sah sich der Vogt der Herrschaft Brandis 75 gezwungen, in die allgemeine Erschließung des Schachens ordnend einzugreifen. Er ließ das Weidrecht der Bauern im Schachen unangetastet und übergab ihnen die Einschläge zur freien Nutzung. Gleichzeitig ordnete er die Zinsverpflichtung, der auch die Schachenleute unterworfen waren. Beiden Parteien oblag die Pflicht, die Schwellen des Emmeufers zu unterhalten, was aber nicht oder nur ungenügend erfüllt wurde. So mußte 1618 der Landvogt David Schöni die Schwellpflicht neu umschreiben. Die Bauern verweigerten aber die Mitarbeit, da sie sich durch das Anwachsen der Schachensiedlung im Recht des Weidganges geschmälert fühlten. 1658 wurden die Bauern gegen die Aufgabe des Rechtes zum Weidgang aus der Schwellenpflicht entlassen ${ }^{76}$. Die Schachensiedlung entwickelte sich unterdessen rasch weiter und zählte 1669 bereits 26 Häuser 77 . Als weitere Gewerbe waren inzwischen eine Gerberei, eine Schmiede, eine Mühle, eine Stampfe und eine Schleife entstanden78. Durch den Weidgang wurde der Schachenwald übernutzt, so daß der Uferschutz nicht mehr sichergestellt war. Daher veranlaßten die Schachenleute 1710 den Landvogt Emanuel Fellenberg, ihre Rechte und Pflichten neu zu regeln. Der Vertreter der Obrigkeit schied zunächst am Emmeufer durch Einzäunen 24 Jucharten geschädigten Waldes aus der Weidezone aus. Hier sollte wiederum Wald aufwachsen, damit Schwellenholz geschlagen werden konnte. Der rest- 
liche Schachen im Halte von etwa 12 Jucharten wurde entsprechend der Zahl der Hausväter in 24 Teile gegliedert und ihnen als unveräußerliches Lehen zugesprochen. Für jede Jucharte Ackerland wurde der Bodenzins auf 5 Schilling festgesetzt. Die durch dieses Reglement entstandene Rechtsamegemeinde war zum Schwellen verpflichtet und wurde daher auch Schwellengemeinde genannt. Bereits vorher hatte sie beim obern Winterseigut 3 Jucharten Wald gekauft, dem vorerst das nötige Schwellenholz entnommen werden konnte ${ }^{79}$.

Noch floß die Emme breit dahin, und es war möglich, durch neue Schwellenbauten Land zu gewinnen. So erhielt 1729 die Rechtsamegemeinde das Recht, zum bereits verliehenen Schachenland weitere 5 Jucharten Reisgrund einzuschlagen, der durch ein gleich großes Stück dem Fluß abgerungenes Land ersetzt werden konnte 80 .

Bis 1923 lastete die Schwellpflicht einzig auf den Inhabern der 24 Nutzungs- und Schwellenrechte. Damals wurde durch ein vom bernischen Regierungsrat für den Emmeschwellenbezirk Rüegsauschachen erlassenes Reglement für die Schwellpflicht auf alles im Talboden gelegene Grundeigentum ausgedehnt 81 .

Folgen wir anhand von Figur 8 wiederum der allgemeinen Entwicklung von Rüegsauschachen. Der Brückenbau von 1764 wirkte sich vorersf kaum aus ${ }^{82}$. Die Zahl der Häuser blieb von 1669 bis um 1800 mehr oder weniger konstant und begann dann allmählich zu steigen. Das Gewerbe wurde reichhaltiger und kräftiger und umfaßte nach und nach auch noch weitere Berufe: Maurer, Wagner, Krämer, Drechsler, Küfer, Schneider, Öler, Schuhmacher, Fischer, Gürtler, Glaser, Messerschmied, Hechler, Zimmermann, Steinhauer, Rechenmacher, Eisenhändler, Bäcker und Köhler (78). Aber noch 1857 schilderte Albert Jahn in seiner Chronik des Kantons Bern ${ }^{83}$ das Dorf wie folgt: "... im Rüegsauschachen, dessen Bewohner übrigens zu den dürftigsten in der ganzen Umgebung gehören, herrscht einige Industrie von Kammachern, Messer- und Hohlbohrerschmieden, deren Fabrikate weithin verführt werden.»

Die allgemeine Industrialisierung, der Bahnbau (1881 Eröffnung der Linie Burgdorf-Langnau, 1899 Burgdorf-Thun) und die Emmekorrektion (ab 1884) brachten neue Impulse. Seither ist aus dem armseligen Schachendorf eine kräftige Siedlung erwachsen, die das alte Zentrum Rüegsau weit überflügelt hat. Die Figuren 4 und 8 zeigen, daß die Zunahme der Gemeindebevölkerung bis 1940 auf das starke Wachstum des heutigen Kerns des im übrigen als Hofgemeinde anzusprechenden Rüegsaus zurückzuführen ist. Seither ist der Rückgang der landwirtschaftlichen Bevölkerung des Hofgebietes stärker als das Wachstum der Talsiedlung. Die Typisierung von Rüegsau als "verstädterte Ge-
Fig. 8 Rüegsauschachen: Wohnhäuser und Einwohner

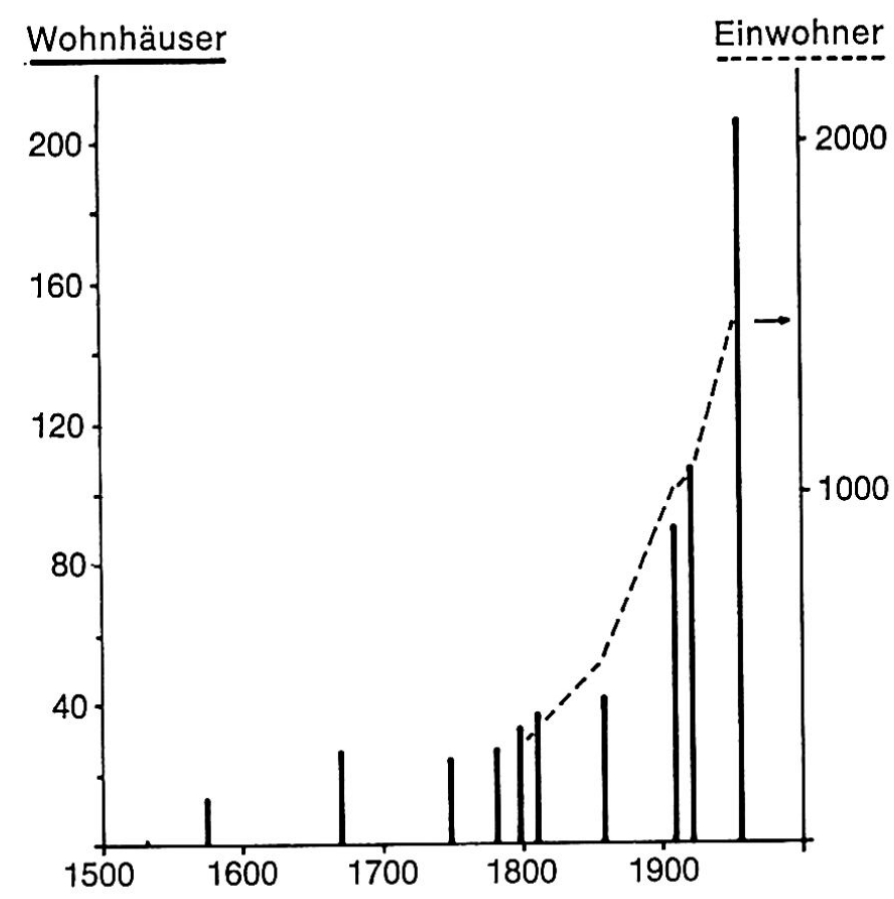

Quellen:

Würgler 1965, 164-169

Eidg. Stat. Amt: Ortschaftenverzeichnisse 1910 u. 1960

meinde mit überwiegender Industriebevölkerung» (Typ F) vermag nicht ganz zu befriedigen, da auf 1221 wohnhafte Berufstätige ein Arbeitsplatzdefizit von 324 Stellen besteht, wovon 267 im sekundären Sektor (siehe Anhang). Immerhin steigt die Zahl der Arbeitsplätze in den Gewerbebetrieben, so daß Rüegsauschachen weiterhin auf Kosten des Hofgebietes an Gewicht gewinnen wird. Der gegenwärtige Ausbau der Verkehrslinien Richtung Burgdorf (Straße, Doppelspur der EBT), das allein schon 148 Arbeitskräfte der Gemeinde Rüegsau absorbiert, dürfte auf die Abwanderung bremsend wirken ${ }^{64}$.

\section{Zusammenfassung}

Abschließend sind die Hauptlinien des Dargestellten herauszuheben. Es ging um Typlandschaften des tieferen und des höheren Mittellandes. Geologische und morphologische Unterschiede wurden festgestellt. Kulturlandschaftlich ließen sich verschiedene Ausbreitungstendenzen erkennen. Von Siedlungen mit gallorömischer Tradition aus begann vom 8. Jahrhundert an die Landnahme im Emmental. Nach einer gewissen Stabilisierung um 1300 setzte um 1500 eine Ausbauphase ein, die zur Besiedlung der Höhenregionen und der bisher gemiedenen Talsohlen führte. Vom Oberemmental her begann seit dem 17. Jahrhundert die Umstellung zu Milchwirt- 
schaft und Käseproduktion. Diese Bewegung erreichte im zweiten Viertel des 19. Jahrhunderts das tiefere Mittelland und bewirkte dort in wenigen Jahrzehnten die Umwandlung der bereits modifizierten Dreifelderwirtschaft zur Kleegraswirtschaft. Die Mechanisierung der Landwirtschaft in der Nachkriegszeit begann im tieferen Mittelland, ermöglichte die Abwanderung von landwirtschaftlichen Arbeitskräften und erhöhte das Einkommen der in der Landwirtschaft Verbleibenden. Von die- ser Entwicklung konnte die Landwirtschaft des höheren Mittellandes nur wenig profitieren, da dort die Möglichkeiten der maschinellen Bebauung beschränkt sind. Daher öffnet sich heute zwischen Talund Berggebiet eine Einkommensschere, die in den höheren Lagen zu einem Rückgang der Bewirtschaftungsintensität und schließlich zu einer $\mathrm{Zu}$ nahme des Waldes führen wird, falls der Berglandwirtschaft nicht außerbetriebliche Mittel zugeführt werden können.

Anhang

Arbeitsplatzbilanz (1960) und Steuerkraft (1963)

\begin{tabular}{|c|c|c|c|c|c|c|c|c|c|}
\hline & 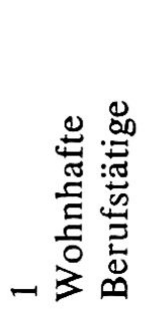 & 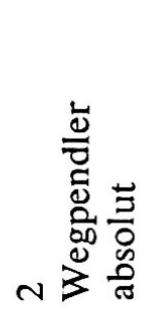 & 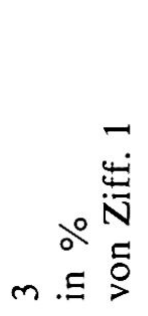 & 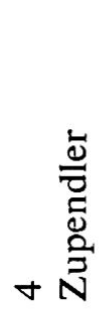 & 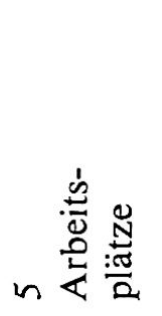 & $6 \frac{N}{\stackrel{N}{=}}$ & 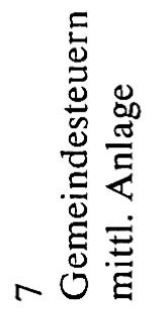 & 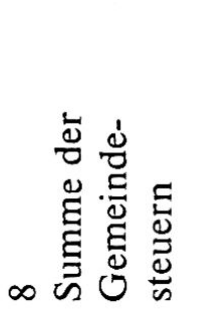 & 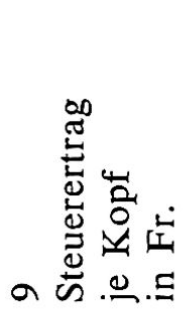 \\
\hline Dotzigen & 351 & 188 & 53,5 & 26 & 189 & -162 & 2,99 & 182678 & 210 \\
\hline Grafenried & 290 & 126 & 43,4 & 3 & 167 & -123 & 3,23 & 182748 & 265 \\
\hline Heimiswil & 835 & 246 & 29,4 & 14 & 603 & -232 & 3,57 & 348236 & 179 \\
\hline Rüegsau & 1221 & 417 & 34,1 & 93 & 897 & -324 & 2,97 & 687316 & 244 \\
\hline Trachselwald & 497 & 70 & 14 & 13 & 440 & -57 & 3,57 & 173821 & 137 \\
\hline Schangnau & 414 & 7 & 1,7 & 3 & 410 & -4 & 3,42 & 124091 & 120 \\
\hline
\end{tabular}

Quellen:

Ziff. 1-6 nach VZ 1960, Q364

Ziff. 7-9 nach Gemeindesteuern Bern 1963

\section{Anmerkungen}

1 Grafenried 1942.

2 Tschumi 1953, $255 \mathrm{f}$.

3 Moosbrugger 1967, 201; Zinsli 1964, 262.

4 HBLS III, 233 und 626.

5 Feller II 342, id. III 144; Häusler 1968, 24 ff.

6 Pulver 1956, 96f.: Meyer 1967, 179; früheste Abweichungen vom Dreizelgensystem konnte Häusler (1968, 48) bereits für das 15. Jahrhundert nachweisen.

7 Feller III 532; Häusler 1968, 43 ff.

8 zitiert nach Feller III 527 f.; vgl. ferner Roth 1948, 33 und Pulver 1956, 97; allgemein zur Ökonom. Ges. siehe Guggisberg und Wahlen 1958.

9 Roth 1948, 12-25; Bodmer 1951, 588.

10 Roth 1948, 25-28.

11 Roth 1948, 34.

72 Roth 1948, 34 und 41.

13 Aerni 1961, 222 f.; detaillierte Zusammenstellung bei Roth 1948, 190 ff.; Pulver 1956, 112.

14 Roth 1948, 191.

15 siehe Schreiben von Schultheiß und Rät der Stadt

Bern an die Amtleute zu Aarberg, Nidau, Erlach, Laupen, Landshut und Zollikofen vom 18. März

1523 (SSRQ Bern 9, 1. Band $341 \mathrm{f} \mathrm{N} \mathrm{155),} \mathrm{wonach}$ die Eichenwälder durch Entnanme von Bauholz allenthalben verwüstet seien und jeder Rechtsamebesitzer verpflichtet sei, jährlich zwei neue Eichen zu setzen und einzuzäumen, damit diese «durch das vych ungeschedigt mogen belyben».

16 Pulver 1956, 27 f.; Meyer 1967, 179 und 269; SSRQ Bern 9, 2. Hälfte 829 ff.

17 Meyer 1967, 154, 160, 179, 269.

18 Meyer 1967, 269.

19 Meyer 1967, 166.

20 Zryd 1942, Vergleich der Tafeln 1749 und 1876;

Meyer 1967, $142 \mathrm{f}$. und $247 \mathrm{f}$.

21 Meyer 1967, 166.

22 Meyer 1967, 163.

23 Sollberger 1968, 13.

$24 \mathrm{vgl}$. Fueter 1928, $152 \mathrm{f}$.

25 Industriestandorte 1967, 52.

26 Volkszählung 1960, Maschinentabellen 8a und b.

27 Heimatbuch Burgdorf 1930 und 1938; Aerni

1965; Heimiswil 1967.

28 Heimiswil 1967, 43-186.

29 Heimiswil 1967, $52 \mathrm{f}$.

30 Heimiswil 1967, 49 f.

31 Heimiswil 1967, 67. 
32 Häusler 1968, 24; vgl. dazu Glässer 1969, 165. 33 nach Heimiswil 1967, 149 ff.; dargestellt in Flatt 1969, 18, Fig. 2.

34 vgl. die Überlegungen von Häusler 1969, 54.

35 Heimiswil 1967, 67.

36 Heimiswil 1967, 86-93.

37 Häusler 1968, 37.

38 Aerni 1965.

39 Wohnbevölkerung Kanton Bern 1968.

40 Gutersohn 1950, 9; id. 1968, 212 ff.

41 LK 1147 und 1148; Hantke 1968, 9.

42 Häusler 1968, 1-23.

43 Feller I 240; Häusler 1958, 22.

44 Häusler 1968, 70.

45 Häusler 1968, 201.

46 vgl. Ramseyer 1961, 28.

47 Häusler 1958, 23.

48 Häusler 1958, 205.

49 Häusler 1968, 78.

50 Sumiswald-Urbar 1572, f. 236.

51 Häusler 1968, 202.

52 weitere Beispiele Pulver 1956, 73 und Häusler 1968. 202.

53 Häusler 1968, 56.

54 siehe Pulver 1956, $67 \mathrm{f}$.

55 Häusler 1968, 53.

56 zum Ägertenhof siehe Pulver 1956, 68 f.; Häusler 1968, $57 \mathrm{f}$.

57 Häusler 1958, 201.

58 Häusler 1968, $60 \mathrm{f}$.

59 Ramseyer 1961.

60 Roth 1958; id. 1966; Häusler 1968, 87 f.

61 Darstellung der Zunahme der Waldfläche bei Gutersohn 1950, 25 und 1968, 215; Häusler (1958, 214 und 322) stellte beim Vergleich der Wald-Etats von 1752/1754 mit den gegenwärtigen Waldarealen fest, daß die heutige Waldfläche gegenüber der Zeit vor 200 Jahren in der Gemeinde Langnau viermal, in Trubschachen achtmal, und in Trub sogar fünfzehnmal größer ist!

62 Gegenüber dem schweizerischen Mittel besteht in der Alterskategorie von 15 bis 59 Jahren eine Lücke von 64 Frauen und 42 Männern (Q 364)..

63 Gerber 1967, 4 ff.

64 Häusler 1968, $211 \mathrm{ff}$.

65 Häusler 1968, $220 \mathrm{f}$.

66 Häusler 1968, 219.

67 Würgler 1965, 165.

68 über die Schachenleute siehe Würgler 1965, 169 f.; Häusler 1968, 247.

69 Häusler 1968, 212.

70 Häusler 1968, 222, vergleiche das Zitat aus dem Sumsiwalder Urbar im Abschnitt 3 (Heimisbach). 71 siehe "Schwellenordnung für das Amt Trachselwald" vom 1. Februar 1766 in SSRQ Bern 9, 2. Hälfte 481-487 N 203 b. Sie wurde am 7. Mai 1768 auch für die Ämter Burgdorf und Landshut anwendbar erklärt.
72 publiziert in Häusler 1968, 230 und $308 \mathrm{f}$.

73 siehe Huber-Renfer 1940, $126 \mathrm{f}$.

74 Würgler 1965, 163-166.

75 Würgler 1949, 11.

76 Würgler 1965, 166.

77 Häusler 1968. 234.

78 Würgler 1965, 171.

79 Würgler 1965, 167; Häusler 1968, 235.

80 Häusler 1968, 235.

81 Würgler 1965, $167 \mathrm{f}$.

82 Marti 1958, 102.

83 Jahn 1857, 618.

84 Q 383.

\section{Literatur}

Karten:: LK $1: 25$ 000, Nrn. 1147 (1954), 1148 (1955), 1167 (1963), 1168 (1963); TA $1: 25000$, Nrn. 142 (1880), 145 (1871), 196 (1885), 368 (1884); Geol. Atlas der Schweiz 1: 25 000, Blatt 22, Kümmerly \& Frey, Bern 1950; dazu Erläuterungsheft. - Schweizerische Grundbuchvermessung, Übersichtsplan Blatt 145, Burgdorf (1965). - Messmer: "Plan von einem Teil der Kirchgemeinden Lützelflüh und Rüexau», undatiert, Anfang des 19. Jhs.; Staatsarchiv Bern, AA IV, Nr. 1508.

Ungedruckte Quellen: Eidgenössisches Statistisches Amt: a) Volkszählung (VZ) 1930, Mappe 374; VZ 1941, Mappe 246; VZ 1950, Mappen 237 und 238; b) $V Z$ 1960, Angaben über Pendler, Maschinentabellen 8a und b und 9a und b; c) VZ 1960, Maschinentabellen, Gliederung der Bevölkerung in Altersstufen zu 5 Jahren; ganzes Material auf dem Eidg. Stat. Amt, Holzikofenweg 8, Bern. - Grafenried, Heimat-Album der Gemeinde, deponiert auf der Gemeindeschreiberei Grafenried, 1942. - Landwirtschaftlicher Produktionskataster der Gemeinden (PK); aufgenommen durch das Eidg. Kriegsernährungsamt, ergänzt durch Neuaufnahmen des Eidg.Volkswirtschaftsdepartementes, Abteilung für Landwirtschaft; deponiert auf der bernischen Zentralstelle für Ackerbau, Bern; daraus entnommen die Angaben über die Getreideproduktion der Jahre 1885, 1919 und 1940. - Sumiswald-Urbar 1572, Staatsarchiv Bern, Urbarien Amt Trachselwald Nr. 27.

Gedruckte Quellen: Eidg. Stat. Amt: Volkszählungen: VZ 1910, L 212; VZ 1960, Q 364 und 383. Landwirtschaft: a) Betriebsstrukturen Q 31 (1929), Q 151 (1939), Q 319 (1955), Q 404 (1965); b) Getreideproduktion L 208 (1917), Q 14 (1929), Q 72 (1934), Q 134 (1939, 1941 und 1942), Q 217 (1943 und 1947), Q 240 (1950), Q 307 (1955), Q 344 (1960), Q 404 (1965). Gewerbe: Q 318 (1955), Q 412 (1965). Siedlung: Schweizerisches Ortschaftenverzeichnis 1910; Francke, Bern 1920. - Schweizerisches Ortschaftenverzeichnis 1960; Bern 1965. 
- Historisch-Biographisches Lexikon der Schweiz (HBLS): 7 Bände, Attinger Neuenburg 1921-1934. - ORL-Institut an der ETH: Industriestandorte, Untersuchung der räumlichen Ordnung der Industrie in der Schweiz, Gesamtbericht; Zürich 1967. Milchverband Bern (MV), Jahresberichte ab 1934/ 1935; Angaben nach Käsereigenossenschaften gegliedert. - Sammlung schweizerischer Rechtsquellen, Kanton Bern, I. Teil: Stadtrechte, Band 9, 1. und 2. Hälfte (SSRQ, Bern 9, 1 und 2), bearbeitet von H. Rennefahrt; Sauerländer, Aarau 1967. Statistisches Büro des Kantons Bern: a) Statistik der Gemeindesteuern im Kanton Bern für 1963; Sonderheft Nr. 6; Bern 1965 b) Die Wohnbevölkerung des Kantons Bern Ende 1968; Beiträge zur Statistik des Kantons Bern, Bevölkerungsstatistik (Reihe A), Heft 2; Bern 1969.

\section{Darstellungen}

Aerni K.: Die Amtsberichte der bernischen Statthalter im 19. Jahrhundert; Archiv des Histor. Vereins des Kantons Bern, 46/1961, Heft 1, 135-252, Bern 1961.

Aerni K.: Zur Entvölkerung der Gemeinde Heimiswil; Geographica Helvetica 20/1965, 132-148, Bern 1965.

Binggeli V.: Simon Gfeller; Francke, Bern 1968.

Bodmer W.: Tendenzen der Wirtschaftspolitik der eidgenössischen Orte im Zeitalter des Merkantilismus; Schweiz. Zeitschrift für Geschichte 1/1951, 562-598.

Feller R.: Geschichte Berns; Lang, Bern; Band I 19633, Band II 1953, Band III 1955.

Flatt K. H.: Die Errichtung der bernischen Landeshoheit über den Oberaargau; Archiv des Hist. Vereins des Kantons Bern, 53/1969, 1_413, Bern 1969. Fueter E.: Die Schweiz seit 1848; Orell-Füßli, Zürich 1928.

Gerber E.: Die Flußauen in der schweizerischen Kulturlandschaft; Geographica Helvetica 22/1967, 1-26, Bern 1967.

Glässer E.: Die ländlichen Siedlungen - Ein Bericht zum Stand der siedlungsgeographischen Forschung; Geographische Rundschau 21/1969, Heft 5, 161-170, Braunschweig 1969.

Guggisberg K., Wahlen H.: Kundige Aussaat köstliche Frucht. Zweihundert Jahre Ökonomische und gemeinnützige Gesellschaft des Kantons Bern, 1759-1959; Verbandsdruckerei, Bern 1958.

Gutersohn H.: Landschaften der Schweiz; Büchergilde Gutenberg, Zürich 1950.

Gutersohn H.: Geographie der Schweiz, Band III, 1. Teil; Kümmerly \& Frey, Bern 1968.

Hantke R.: Allgemeines zur Erd- und Vegetationsgeschichte (der Schweiz); Ur- und frühgeschichtliche Archäologie der Schweiz, Band I; Schweiz. Ges. für Ur- und Frühgeschichte, Basel 1968.
Häusler F.: Das Emmental im Staate Bern bis 1798; Schriften der Berner Burgerbibliothek, 2 Bände; Bern 1958 und 1968.

Heimatbuch des Amtes Burgdorf; 2 Bände; Langlois, Burgdorf 1930 und 1938.

Heimiswil - Heimatbuch einer bernischen Landgemeinde; Haller \& Jenzer, Burgdorf 1967.

Huber-Renfer F.: Die Emme und ihre Ufer; Burgdorfer Jahrbuch 8/1941, 71-148; Langlois, Burgdorf 1940.

Jahn A.: Chronik des Kantons Bern; Stämpfli, Bern 1857.

Marti W.: Die Holzbrücke von Hasle-Rüegsau; Burgdorfer Jahrbuch 26/1959, 93-134; LangloisBurgdorf 1958.

Meyer K. A.: Holzarten und früherer Forstbetrieb im «bernischen» Mittelland; Mitteilungen der Schweiz. Anstalt für das forstl. Versuchswesen, Birmenstorf, 43/1967, Heft 2, 71-287; Beer \& Cie, Zürich 1967.

Moosbrugger R.: Die frühmittelalterlichen Gürtelbeschläge der Schweiz; Band 14 der Monographien zur Ur- und Frühgeschichte der Schweiz; Birkhäuser, Basel 1967.

Pulver E.: Von der Dreizelgenordnung zur bernischen Kleegraswirtschaft; Zürich 1956.

Ramseyer R.: Das altbernische Küherwesen; Sprache und Dichtung. Neue Folge Band 8; Haupt, Bern 1961.

Roth A.G.: G. Roth \& Co. AG Burgdorf 18481948, Gedenkschrift über 100 Jahre einer Exportfirma für Emmentaler Käse; Burgdorf 1948.

Roth A. G.: Vom Küher zum Käser; Chronik des Amtes Fraubrunnen 3/1958, 35-45; hg. vom Ökonomischen und Gemeinnützigen Verein des Amtes Fraubrunnen.

Roth A.G.: Aus der Geschichte des Emmentaler Käses; Sonderdruck aus "75 Jahre Emmentalischer Käserverein» 1963; Haller \& Jenzer, Burgdorf 1966. (Sollberger M.): Waldbauliche Auswirkungen der Privatwald-Zusammenlegungen Grafenried; Diplomarbeit ETH, Manuskript (1968).

Tschumi O.: Urgeschichte des Kantons Bern; Huber, Bern 1953.

Würgler H.: Die Entstehung des Amtes Trachselwald; Quellenhefte zur Geschichte und Heimatkunden des Amtes Trachselwald, Heft 1; Haupt, Bern 1949.

Würgler H.: Heimatkunde von Rüegsau; hg. von der Einwohnergemeinde Rüegsau; Fischer, Münsingen 1965.

Zinsli P.: Namenkundliches zum Deutschwerden der schweizerischen Alpentäler; Alemannisches Jahrbuch 1962/63 255-282; Schauenburg, Lahr 1964.

Zryd P.: Grafenried zur Zeit der Dreifelderwirtschaft; Francke, Bern 1942. 